\title{
Passive Localization Processing for Tactical Unattended Ground Sensors
}

Lawrence C. $\mathrm{Ng}$

Eric F. Breitfeller

RECEIVED

DEC 271995

OSTI

September 1995
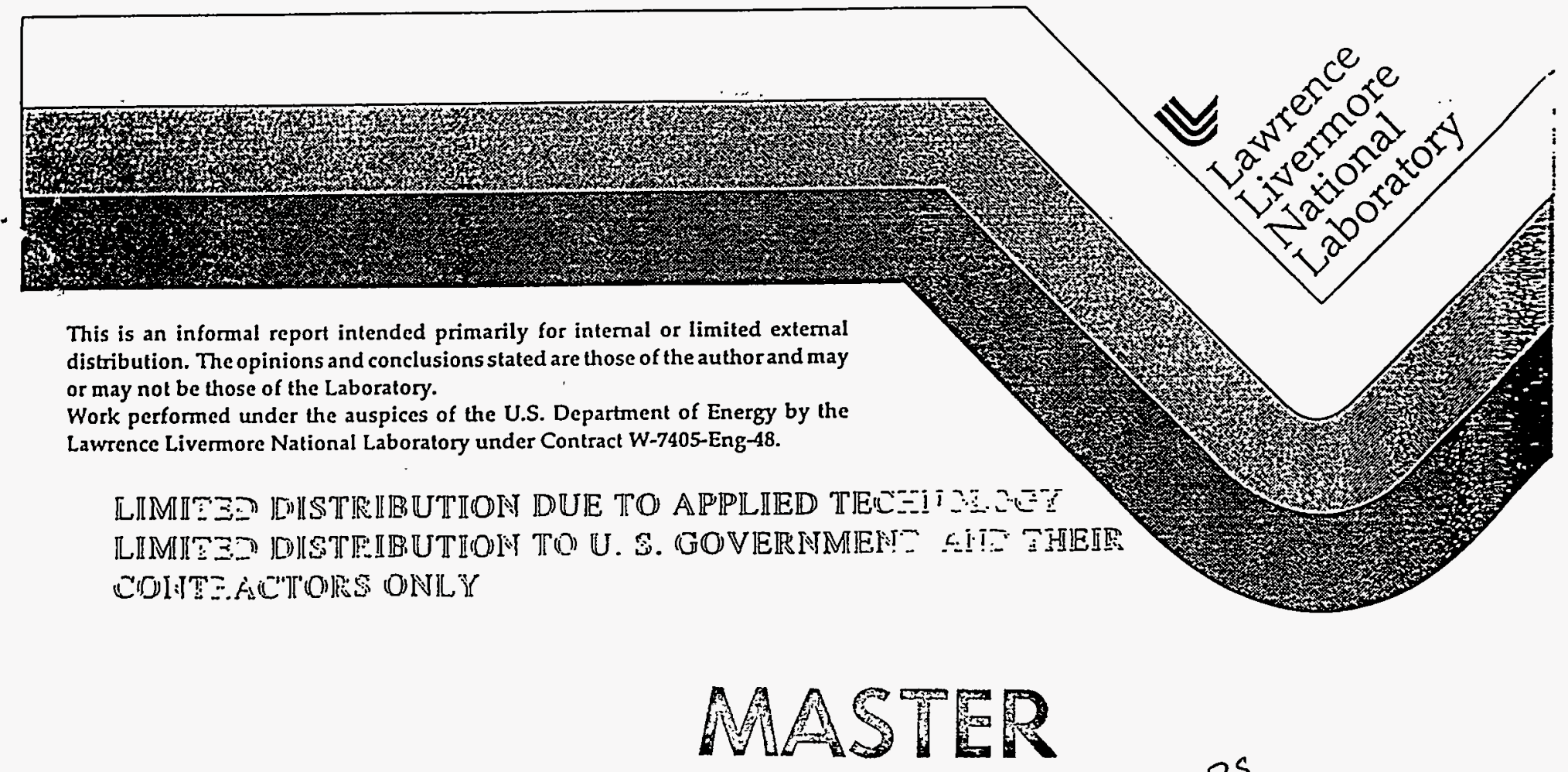

DISTRIBUTION OF THIS DOCUMENT IS UNLRMITEDO 35 
This dosument was prepured as an account of work sponsored by an agencf of the United Slates Govornment Neither the Linitod Suter Government nor the University of Californiz nor any of their employees, makes any warrant5, expros or inplied, or assumes ang legal lizbility or responsibility for the accuracy, complecenes, or usefulness of ans information, apparalus, produch, or proces disclosed, or represents that its use would not infringe privaled ouned righes. Reference herein to any specific commercial products, process, or service by trade name, trademarti anulacturor, or otherwise, does nol necossarily constitute or imply its endorsement, recommendation, or fe voring by the Uniled States Government or the University of Catifornia. The views and opinions of authors coprosed herein do not necosarily state or reflect those of the Uniled States Government or the University of Calforniz, and shall not be used for advertising or product endorsement purposes.

This report has been reproduced directly from the best a vailable copy.

A vailable to DOE and DOE contractors from the Orice of Scientific and Technical Information

$$
\text { P.O. Box 62, O2k Ridge, TN } 37831
$$

Prices 2 railable from (615) 576-8401, FTS 626-8401

Available to the public from the

National Technical Information Service

US. Departmest of Commerce

5285 Port Royal Rd,

Springlield, VA 22161 


\begin{abstract}
This report summarizes our preliminary results of a development effort to assess the potential capability of a system of unattended ground sensors to detect, classify, and localize underground sources. This report also discusses the pertinent signal processing methodologies, demonstrates the approach with computer simulations, and validates the simulations with experimental data. Specific localization methods discussed include triangulation and measurement of time difference of arrival from multiple sensor arrays.
\end{abstract}

Administrative Information*

This effort was supported by NAI directorate's TUGS project, led by Dr. Steve Peglow of $D$ division. Both authors are with the Engineering Research Division of the Electronics Engineering Department. Dr. Lawrence Ng is the Group Leader of the Signal and Image Processing Research Group and Eric Breitfeller is also a member of the same group.

*Work performed under the auspices of the U.S. Department of Energy by Lawrence Livermore National Laboratory under contract W-7405-Eng-48. 
Table of Contents

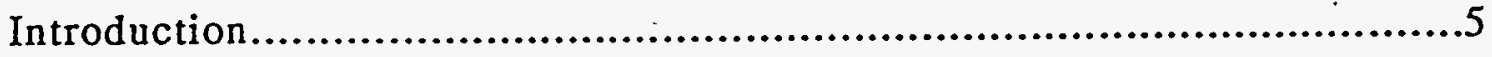

Description of the Problem ........................................................5

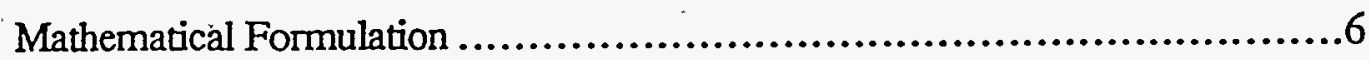

Localization Via Triangulation...............................................................

Beamforming...............................................................

Direction Finding ...........................................................

Performance Prediction ..................................................11

Localization Via Measurement of TDOA ...........................................11

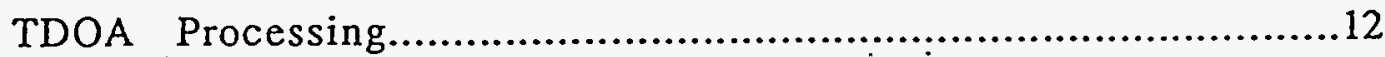

Source Localization . Estimation.....................................................13

Performance Prediction .................................................... 14

Simulation Studies............................................................. 14

Analysis of LLNL onsite Acoustic Experiments ..................................17

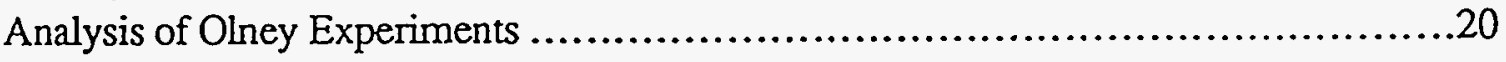

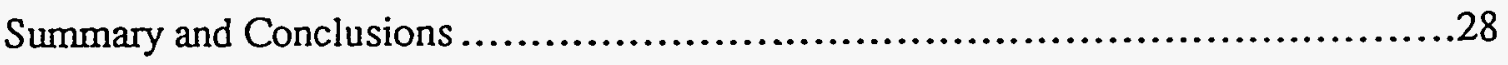

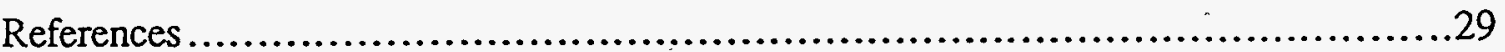


Figure 3 A generalized beamformer for array processing $. . \ldots \ldots \ldots \ldots \ldots \ldots \ldots \ldots \ldots \ldots \ldots . . . .19$

Figure 4 Beam interpolation for fine measurement ....................................10

Figure 5 Range error versus bearing accuracy .......................................11

Figure 6 TDOA localization from three sensor arrays...................................12

Figure 7 A typical two channel correlator...............................................13

Figure 8 Position error for a simulation.................................................14

Figure 9 TDOA estimation error versus SNR.......................................14

Figure 10 Beamforming simulation with an eight element circular array

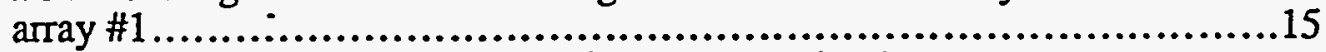

Figure 11 Beamforming simulation with an eight element circular array

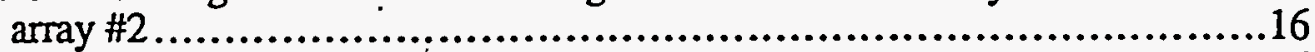

Figure 12 Two sensor arrays correctly .................................................16

Figure 13 Superposition of simulated beam.............................................16

Figure 14 Schematic map of LLNL acoustic experiment site ...........................18

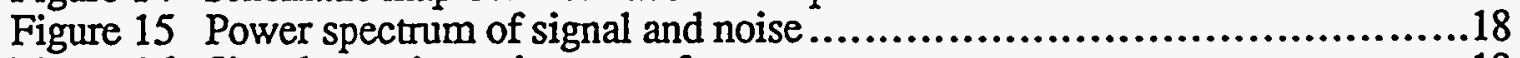

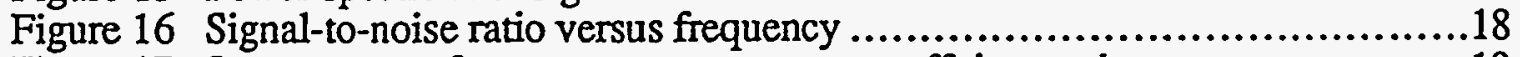

Figure 17 Spectrogram of sensor output near source off time at the $\ldots . . . \ldots \ldots \ldots \ldots \ldots . . .19$

Figure 18 Beam pattern from a linear array ............................................19

Figure 19 Beam pattern from a circular array .......................................19

Table 1 Olney circular array surveyed locations in a right-handed coordinate system.....................................................................20

Figure 20 Map of Olney experiment showing sensor placements and underground structure...........................................................21

Figure 21 Full spectrogram of a single channel data from experiment af2-1ch0..........22

Figure 22 Cut spectrogram of a single channel data from experiment af $2-1$ ch0 $\ldots . . . \ldots .22$

Figure 23 Cut spectrogram of a full beam data from experiment af $2-1 \ldots \ldots \ldots \ldots \ldots \ldots . .23$

Figure 24 Signal detection from a single channel........................................23

Figure 25 Multisensor correlation from experiment af $2-1 . \ldots \ldots \ldots \ldots \ldots \ldots \ldots \ldots \ldots \ldots \ldots \ldots . . .24$

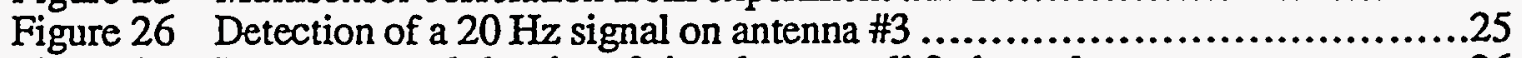

Figure 27 Power spectral density of signal across all 8 channels.......................26

Figure 28 Signal levels across all channels for antenna \#3 .............................27

Figure 29a Beam pattern for antenna \#2......................................................27

Figure $29 \mathrm{~b}$ Beam pattern for antenna $\# 3$................................................27.

Figure 30 Beamforming results from Olney data and superposition onto ................28 


\section{Introduction}

An important goal of the TUGS (Teamed Unattended Ground Sensors) project is to develop and assess a system's capability to localize underground sources like diesel generators and other machinery that emit continuous acoustic, seismic, or electromagnetic signatures that can be detected from a suite of air deployed sensors [1]. In order to reach this goal, some of the key issues needed to be addressed are: (1) how to process the raw data by individual sensors? (2) what data each sensor needs transmitting? and (3) how to fuse data from same or different type of deployed sensors? and (4) what is the best configuration or geometry for sensor deployment? Answers to these and other questions can be obtained in part by examining the multi-sensor localization approach.

This memorandum summarizes our recent study on multi-sensor localization processing. We describe two different approaches to estimate source location passively. The first method employed two or more directional arrays to localize a source via triangulation. The second method relied on three or more omni or directional arrays to localize a source via measurement of TDOA (Time difference of arrival). Perhaps the major distinction between the two approaches is: triangulation method requires beamforming processing while TDOA does not. This difference may be an important consideration since . beamforming requires accurate knowledge of sensor locations with respect to each other.

In this report we examine in length key issues relating to these two approaches. We also describe their technical merits, including signal processing flow, and prediction of performance. We also demonstrate the two approaches with computer simulations, and further validate their applications with experimental data.

\section{Description of the Problem}

Given an underground source like a diesel generator, for example, its operation may be detected by microphones or/and geophones deployed at some distance from the underground structure such as that depicted in Figure 1 . The absolute position of the sensor may be measured and therefore limited by the onboard GPS accuracy of about 6 meters. When operating in a differential GPS mode, the relative positions of sensor with respect to each other may be accurate to about one meter [2].

By processing the signal both locally and globally, we wish to localize the underground sources. Local processing is done locally within each sensor or among sensors within an array. Global processing is done with inputs collected from all sensors or sensor arrays. Obviously an important question taht needs to be addressed is: how much processing should be done locally and globally and their performance trades. However the answer to this question will be dependent on how localization processing is implemented.

In essence two different approaches are available as we have mentioned earlier: Triangulation and TDOA. Triangulation requires a minimum knowledge of two bearings to the source and the baseline distance between sensor arrays. Therefore the sensor array must. have good directionality or spatial filtering capability. This can be accomplished with a beamformer. The second approach requires knowledge of two TDOA measurements from a minimum of three sensor arrays. However, directionality of the sensor array is not used explicitly in the calculation although directionality will provide additional array gain and discrimination capability against interferences. We discuss these two approaches in detail in the next two sections. 


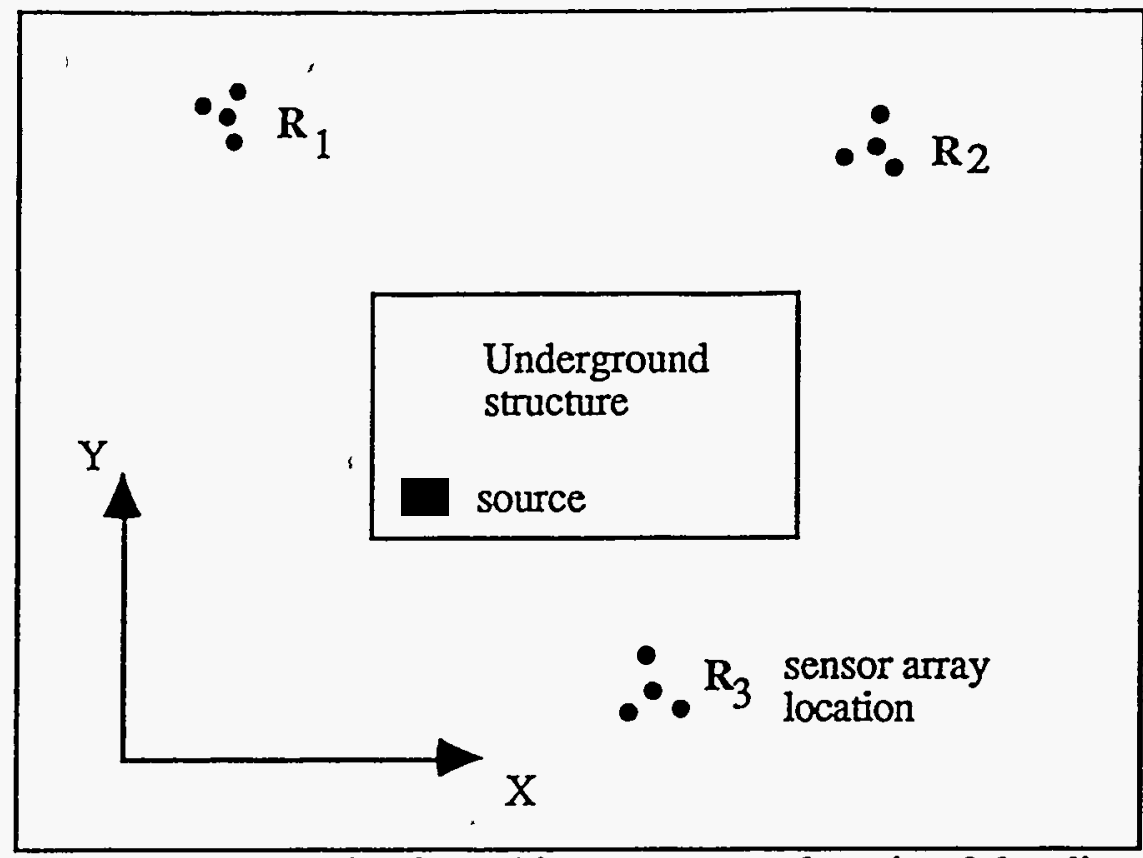

Figure 1 Schematic of a multi-sensor source detection \& localization

\section{Mathematical Formulation}

The source localization problem can be formulated mathematically as follows and for simplicity we will assume a planar problem:

Let the source location be $r_{0}=\left(x_{0}, y_{0}\right)^{t}$, a column vector, whose values we want to estimate. Let $R_{i}, i=1,2 \ldots M$ be the sensor location vector for the $M$ sensor arrays. Thus assuming $\mathrm{N}$ sensors are available within each array we can denote the locations of each sensor by:

$$
R_{i}=\left(r_{i 1}, r_{i 2}, \ldots, r_{i N}\right)^{t}
$$

where each $r_{i n}$ is measured with respect the the array centroid vector $R_{i c}$. For a plane wave arrival assumption, the signal received at each sensor in the ith sensor array can be written compactly ạs:

$$
x_{i}=a_{i} e^{j\left(\omega t+K_{i} \cdot R_{i c}\right)}\left[e^{j K_{i} \cdot R_{i}}\right]+n_{i}
$$

where a is the signal amplitude, $\omega$, source signal frequency, $\mathbf{n}_{\mathbf{i}}$, the $N$ element vector of sensor noise, and $\mathrm{Ki}_{\mathrm{i}}$, the wave number vector with magnitude $\omega / \mathrm{c}$ ( $c$ is the medium propagation speed), and direction $e_{i}$, which is pointing from the ith array centroid to the incoming plane wave. It is understood that the quantity in the bracket is a column vector and can be denoted by 


$$
v_{i}=\left(e^{j \omega\left(e_{i} \cdot r_{i 1}\right) / c}, e^{j \omega\left(e_{i} \cdot r_{i 2}\right) / c}, \ldots, e^{j \omega\left(e_{i} \cdot r_{i N}\right) / \epsilon}\right)^{t}
$$

and is known as the steering vector. Therefore using Eqs.(2) and (3) signal from all sensors can be rewritten as:

$$
x_{i}=a_{i} e^{j\left(\omega+k_{i} \bullet R_{i c}\right)} v_{i}+n_{i} \quad ; \mathrm{i}=1,2, \ldots, \mathrm{M}
$$

\section{Localization Via Triangulation}

Figure 2 shows the basic triangulation geometry. By measuring bearings to target " $\mathrm{C}$ " from sensor arrays " $A$ " and " $B$ " and with knowledge of the intersensor baseline distance "L", target range can be computed easily from:

$$
R=\frac{L \sin \theta_{2}}{\sin \left(\theta_{2}-\theta_{1}\right)}
$$

Triangulation accuracy is usually good for ranges less than 5 times the baseline distance. Using Eq.(5), one can bound the performance of the range estimate from uncertainty of baseline distance and bearing measurements by:

$$
\sigma_{R}=\left(\frac{R}{L}\right) \sqrt{\sigma_{L}^{2}+\left(\frac{\sin ^{2} \theta_{1}+\sin ^{2} \theta_{2} \cos ^{2}\left(\theta_{2}-\theta_{1}\right)}{\sin ^{4} \theta_{2}}\right)\left(R \sigma_{\theta}\right)^{2}}
$$

where $\sigma_{L}^{2}$ is the variance of baseline uncertainty, and $\sigma_{\theta}^{2}$ is the variance of the bearing measurement. Thus, we note that the range uncertainty is linearly proportional to the range to baseline ratio. 


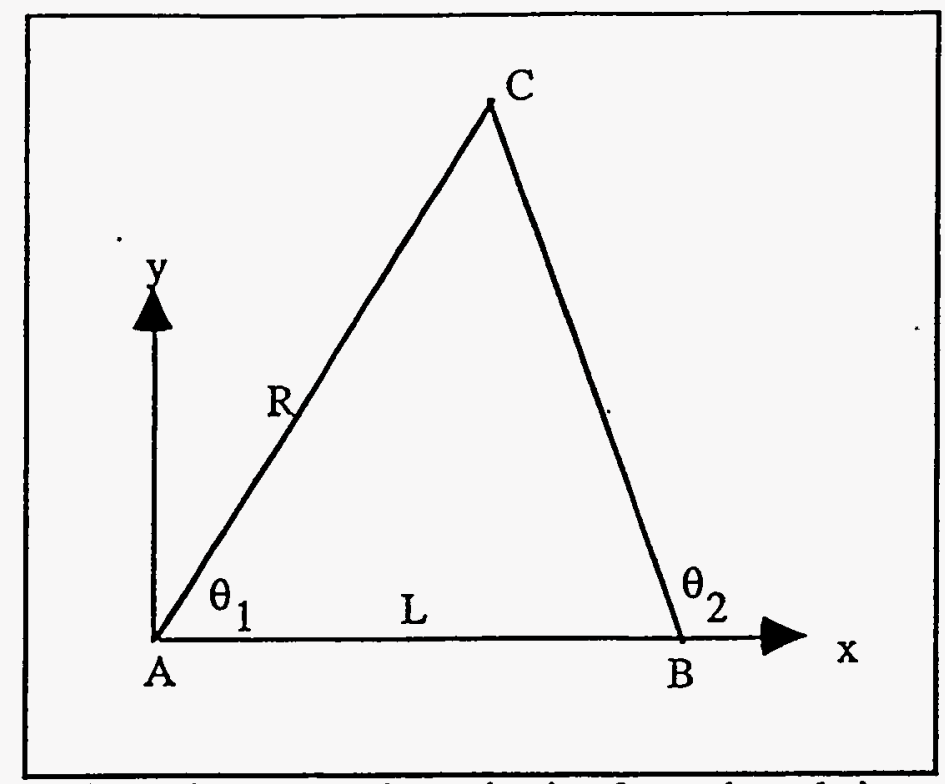

Figure 2 Range determination from triangulation

\section{Beamforming}

Beamforming is a standard technique to find the direction of a signal source. Similar techniques are applicable for processing underwater sonar, seismic, and radar data [3-5]. Figure 3 shows a generic array signal processing diagram of a beamformer. Here signal from all sensors are collected and processed through five different operations: (1) spectral shaping that provides prewhitening (or flattening) of temporal background noise and spectral enhancement filtering, (2) spatial shaping that provides optimal prewhitening of the background spatial noise, (3) phase (or beam) steering that time aligns the signal received at different sensors, (4) summing the time aligned data to achieve signal to noise gain, and finally (5) squaring the data to compute the beam power. The objective of choosing $\mathrm{H}(\omega)$ and $\mathrm{Q}(\omega)$, the spectral and spatial filters, is to maximize the response along the beam steered direction and minimize the response in any other directions. This is equivalent to the design of a narrow band frequency filter. By steering a beam or forming multiple beams over the region of interest, one can detect and identify the direction of the source.

Thus referring to Figure 3, the beam output for a plane wave with frequency $\omega$ is given by:

$$
\begin{aligned}
B(\omega) & =\left|H(\omega) \mathrm{v}^{*}(\omega) Q^{-1}(\omega) \mathrm{x}\right|^{2} \\
& =\left|\mathrm{w}^{*} \mathrm{x}\right|^{2}
\end{aligned}
$$

where ()$^{*}$ denotes the complex conjugate transpose of the quantity and

$$
w^{*}=v^{*}(\omega) Q^{-1}(\omega) H(\omega)
$$


is a complex weighting vector.

For a conventional beamformer, we can assume the sensors are positioned such that they are spatially uncorrelated and therefore the spatial covariance matrix $\mathbf{Q}$ is an identity matrix. The spectral shaping matrix is also a diagonalized matrix with identical filter whose frequency response is given by [3]:

$$
|h(\omega)|^{2}=\frac{S(\omega) / N(\omega)}{1+\nu^{*}(\omega) \nu(\omega) S(\omega) / N(\omega)} \frac{1}{N(\omega)}
$$

where $S(\omega)$ and $N(\omega)$ are the signal and noise power spectral densities. Note that this filter's operations consist of first pre-whitening the noise (last quantity in Eq.(9)) and frequency selection based on the signal-to-noise ratio (SNR). In its simplest form for a case with constant SNR, the frequency selection filter could be a frequency window such that outside of this window the signal power goes to zero. For a conventional beamformer, signal processing on each channel is decoupled.

For advanced beamformers such as the high resolution and maximum entropy beamformers, explicit use of signal and noise covariance matrices are needed [7,8]. In this case the signal processing channels are coupled among sensors.

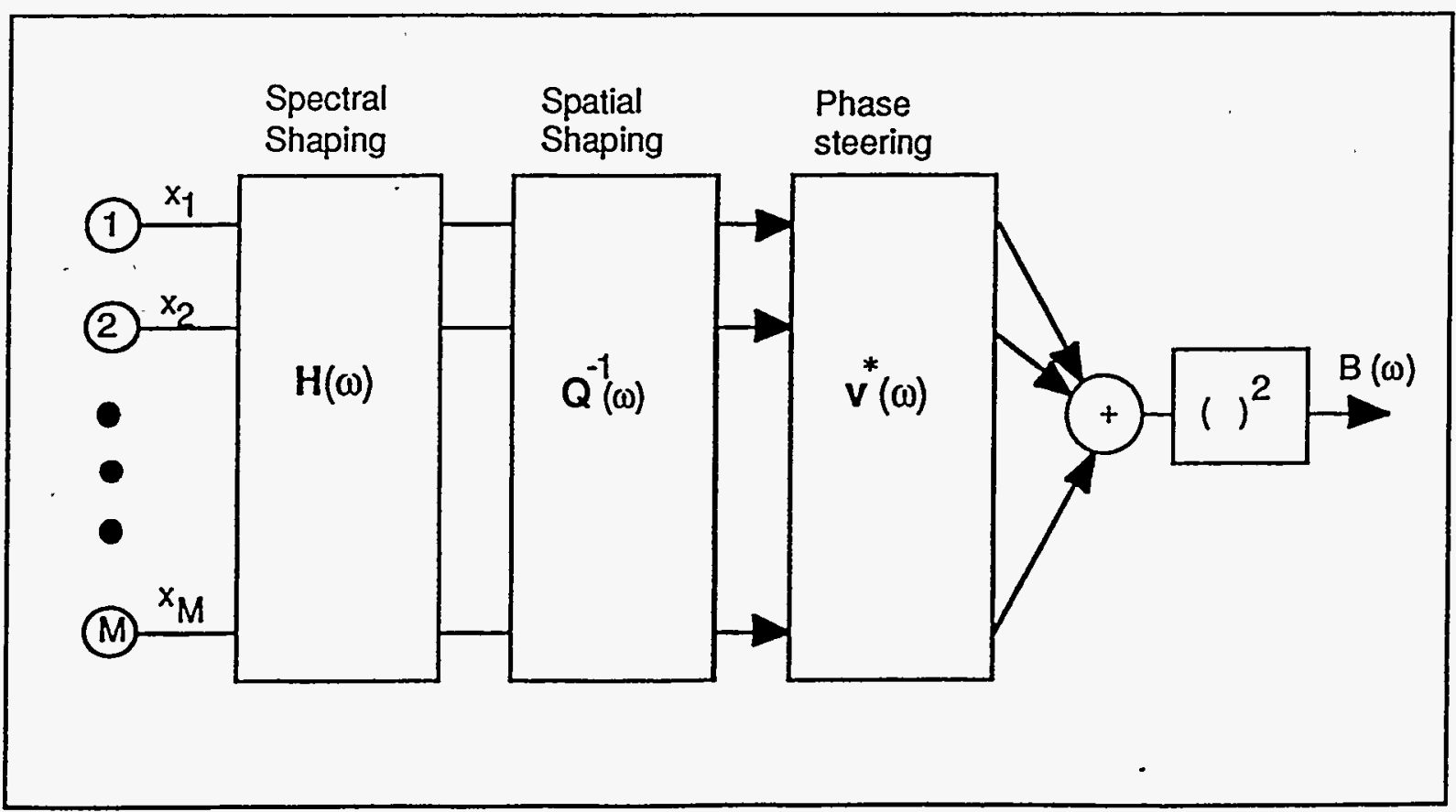

Figure 3 A generalized beamformer for array processing 
Direction Finding

It is a well known fact that for a conventional beamformer, the spatial resolution of an array is determined by its diffraction limit. If $\lambda$ is the wavelength and $L$ is the aperture size, the diffraction limit (or beamwidth) is given by:

$$
\Delta \theta \cong \frac{\lambda}{\cdot \bar{L}}
$$

However in order to obtain a finer estimate, one can steer the array to obtain beam power response within the beam. With a measurement of at least three points, one can obtain a finer estimate by interpolation. Figure 4 shows measurement of three beam power responses within a beam, then using a quadratic interpolation, the estimate of the direction of the maximum response axis (MRA) is given by:

$$
\hat{\theta}=\left(\frac{B_{-}-B_{+}}{B_{-}+B_{+}-2 B_{o}}\right)\left(\frac{\delta \theta}{2}\right)
$$

where $\delta \theta$ is the beam steering spacing.

The accuracy of the estimate, as a function of SNR, is given by [8]:

$$
\sigma_{\theta}=\frac{\Delta \theta}{\sqrt{S N R}} .
$$

Thus for low SNR situation like at $0 \mathrm{~dB}$, the bearing measurement uncertainty is on the order of a beamwidth. On the other hand at high SNR like $20 \mathrm{~dB}$, the measurement is accurate to $10 \%$ of the beamwidth.

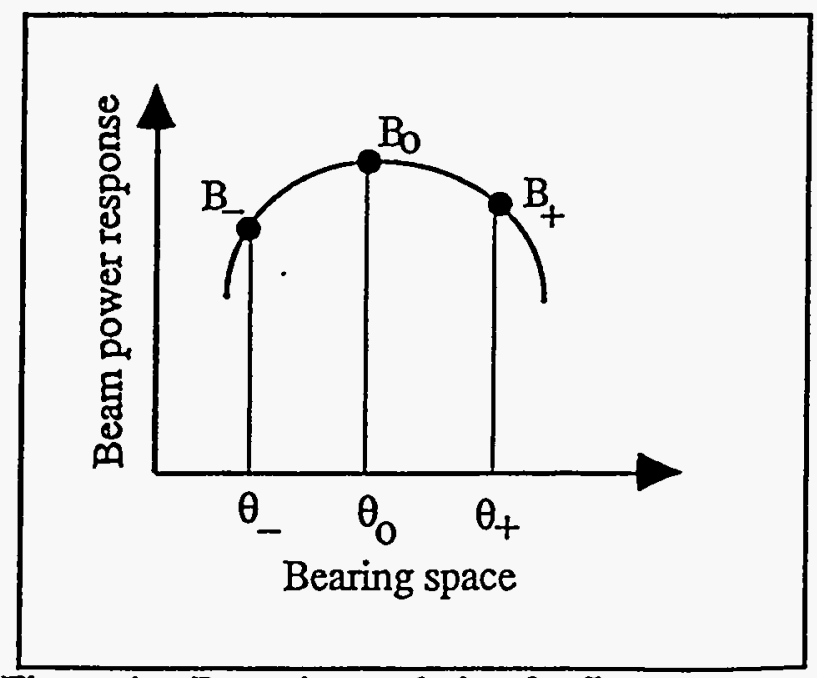

Figure 4 Beam interpolation for fine measurement 


\section{$\because \quad$ Performance Prediction}

Figure 5 shows the prediction of localization performance using Eq.(6) for two sensor arrays with a baseline of $100 \mathrm{~m}$. We assume the source is located equi-distant from the two sensor arrays (See Fig. 2). Note that the lower limits were determined by the uncertainty in the baseline distance estimate. Here we assume a meter accuracy achievable with a different GPS system. Of course this error will be essentially zero for ground experiments where sensor location can be accurately surveyed. Thus, under this set of asșumptions, we note that for a bearing accuracy of $0.1^{\circ}$, we can localize the source at $5^{*} \mathrm{~L}$ or $500 \mathrm{~m}$ to within $10 \mathrm{~m}$. On the other hand, the cross range error is less than $(500 \mathrm{~m}) *(1.7 \mathrm{mrad})=0.85 \mathrm{~m}$. It is typically the case that for passive localization, the range error is an order of magnitude greater than the cross range estimate.

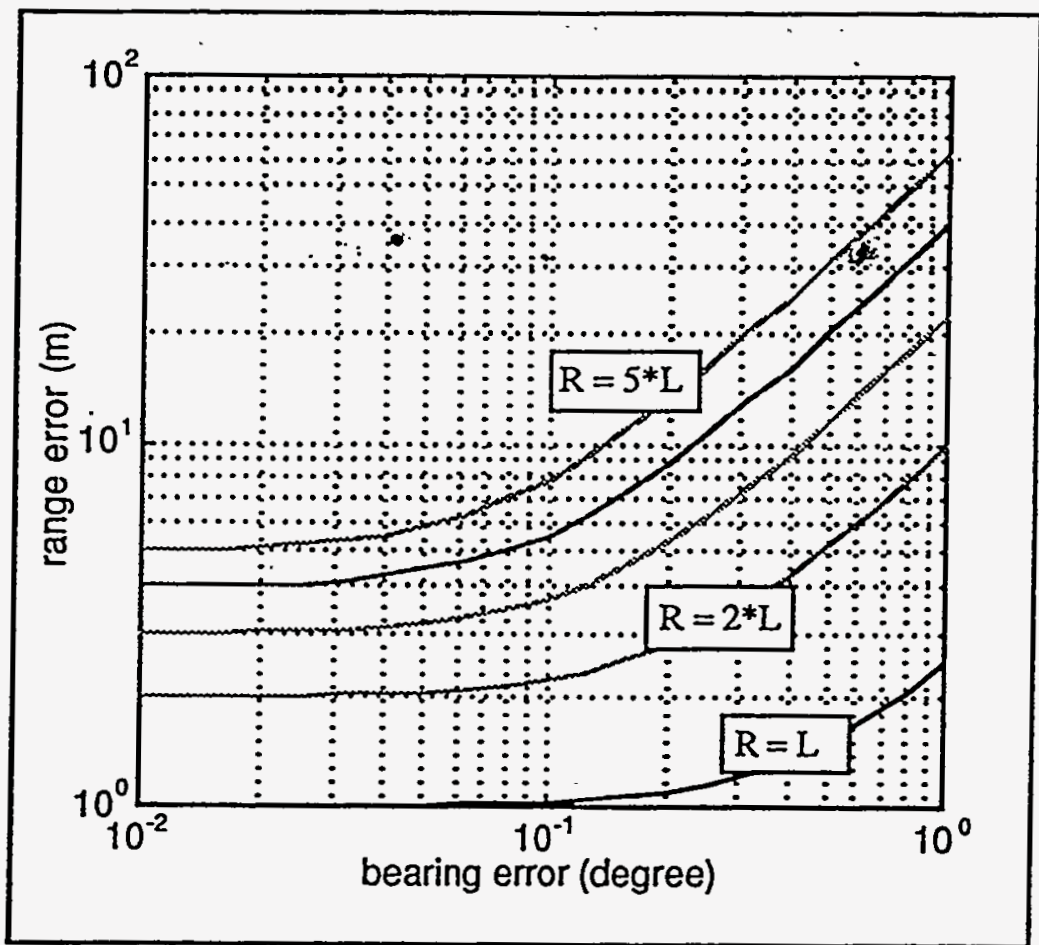

Figure 5 Range error versus bearing accuracy

\section{Localization Via Measurement of TDOA}

It is well known that TDOA measurement from two sensor arrays defines a hyperbolic curve that passes through the signal source $[9,10]$. Thus with a second pair of sensor arrays one can determine the source location at the point of intersection for a planar geometry. It is true that there may be more than one intersection point. However this ambiguity can usually be discriminated by prior knowledge of the approximate source location. Furthermore, by adding another sensor array, one can determine the source position in 3D space. We will briefly examine this approach and assess its performance. 


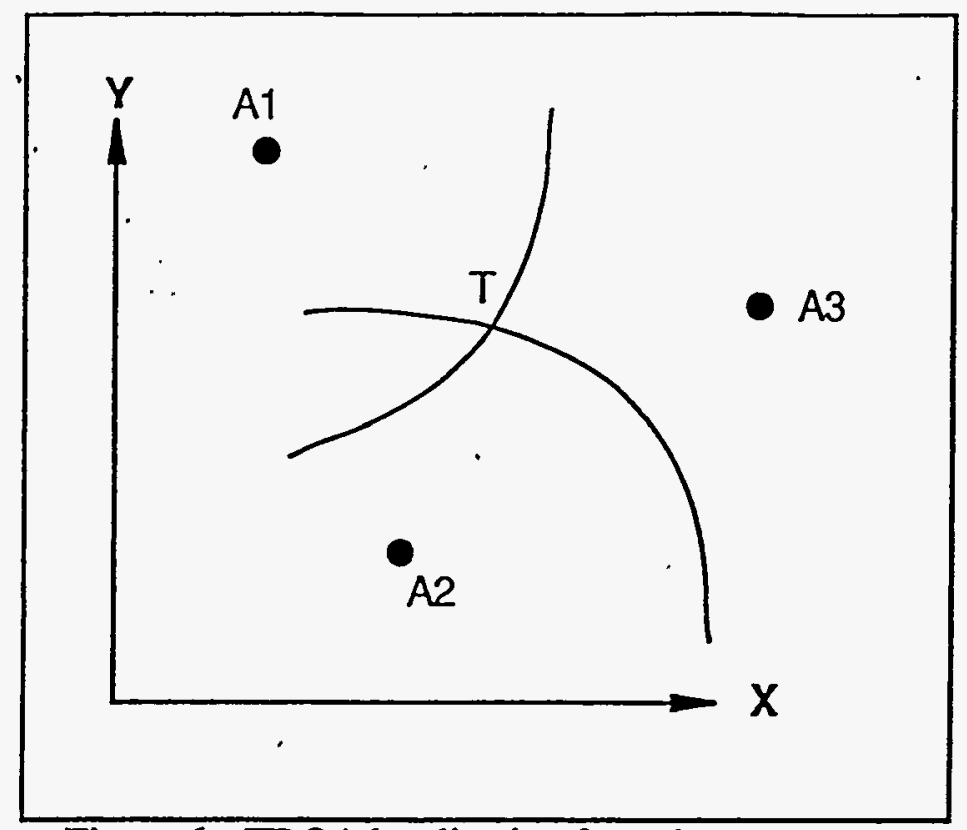

Figure 6 TDOA localization from three sensor arrays

\section{TDQA Processing}

Time delay estimation of a common continuous signal between two channels or arrays can be measured by a correlation receiver. As shown in Figure 7, signal from an array is first shaped by a spectral filter to enhance the SNR. It is then followed by a frequency domain cross-correlator. Taking the inverse FFT of the result yields the desired correlation function. The peak of this correlation function gives an estimate of the desired TDOA.

The uncertainty of the estimated TDOA is given by the Cramer-Rao lower bound expression [9]:

$$
\begin{aligned}
\sigma_{\tau}^{2} & \geq \frac{2 \pi}{2 T \int_{F_{1}}^{F_{2}^{2}}\left[\frac{S^{2}(\omega) / N^{2}(\omega)}{1+2 S^{2}(\omega) / N^{2}(\omega)}\right] \omega^{2} d \omega} \\
& \geq \frac{3 / 8 \pi^{2}}{B T}\left[\frac{1}{F_{2}^{2}+F_{I}^{2}+F_{1} F_{2}}\right]\left[\frac{1+2 S / N}{(S / N)^{2}}\right]
\end{aligned}
$$

where $\mathrm{B}$ is the signal bandwidth, $\mathrm{T}$ is the integration time, and $\mathrm{S} / \mathrm{N}$ is the signal-to-noise ratio and $F_{1}$ and $F_{2}$ are the lower and upper values of the frequency window. 


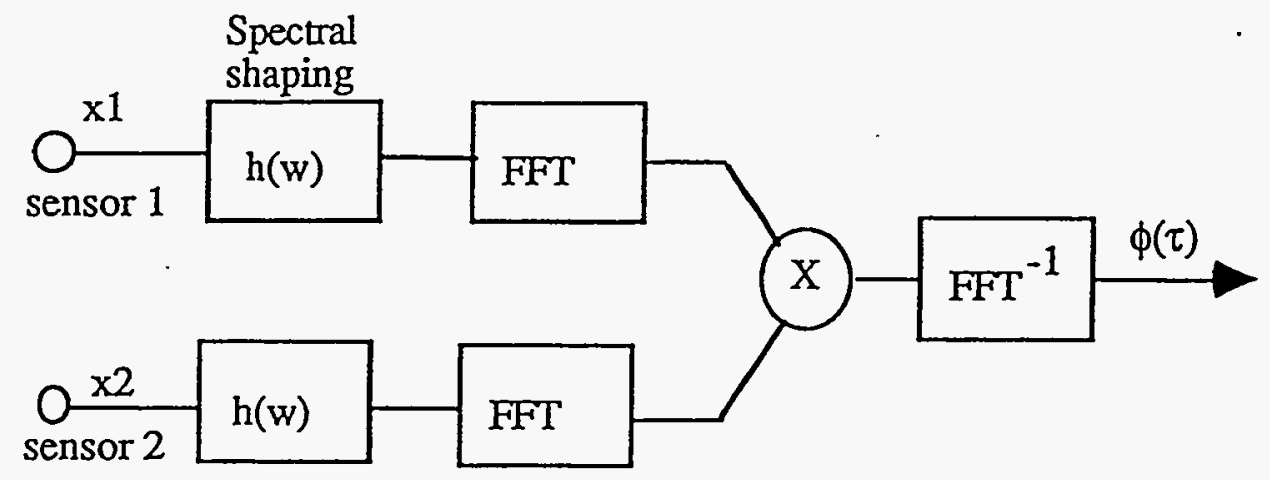

Figure 7 A typical two channel correlator

\section{Source Localization Estimation}

Let the three sensor arrays A1, A2, and A3, as shown in Figure 6, be located at $(\mathrm{x} 1, \mathrm{y} 1),(\mathrm{x} 2, \mathrm{y} 2)$, and $(\mathrm{x} 3, \mathrm{y} 3)$. Furthermore, let the target be at position $(\mathrm{x}, \mathrm{y})$, and its distance to the three sensors be R1, R2, and R3 respectively. Then the two independent TDOA measurements can be written as:

$$
\begin{aligned}
{\left[\begin{array}{l}
\tau_{12} \\
\tau_{23}
\end{array}\right] } & =\left[\begin{array}{l}
f_{1}(x, y)-f_{2}(x, y) \\
f_{2}(x, y)-f_{3}(x, y)
\end{array}\right]+\left[\begin{array}{l}
n_{1} \\
n_{2}
\end{array}\right] \\
& \equiv \boldsymbol{h}(x, y)+n
\end{aligned}
$$

where

and

$$
\tau_{i j}=\left(R_{i}-R_{j}\right) / c ; 1 \leq \mathrm{i} \leq \mathrm{j} \leq 1,
$$

$$
f_{i}(x, y)=\frac{1}{c} \sqrt{\left(x-x_{i}\right)^{2}+\left(y-y_{i}\right)^{2}} .
$$

Note that unlike a short seismic pulse where one can measure the TDOA by detecting arrival time of a pulse, we must, for a continuous source, cross correlate between sensors to find the relative TDOA. The performance of localization given by its covariance matrix can be determined from: 


$$
\operatorname{Cov}(x, y)=\left(H^{t} H\right)^{-1} \sigma_{\tau}^{2} \quad \because
$$

$$
\therefore
$$

where $\mathrm{H}()$ is the Jacobian matrix of $h(x, y)$ evaluated at the true source location, and $\sigma_{\tau}^{2}$ is the variance of the TDOA estimate. The circular error probability (CEP) of the position estimation error is therefore given by:

$$
C E P=0.59\left(\sigma_{x}+\sigma_{y}\right)
$$

\section{Performance Prediction}

Figure 8 shows the localization performance using TDOA calculation from three sensor arrays. We assumed the relative array location is accurate to $3 \mathrm{~m}$. The three sensor $\therefore$ arrays are located at $(0,0)(0,100)$, and $(100,0)$ forming a right triangle. The target is located respectively at $2,4, \ldots, 10$ times the baseline distance and along the triangular array axis of symmetry. At a high SNR of $20 \mathrm{~dB}$, the position enror is less than $5 \mathrm{~m}$ even though the target is at a distance 10 times the base. Figure 9 shows the correspondent TDOA error as a function of SNR. For the TDOA calculation, we assume a $10 \mathrm{~Hz}$ bandwidth signal at $100 \mathrm{~Hz}$ and an integration time of 1 second.

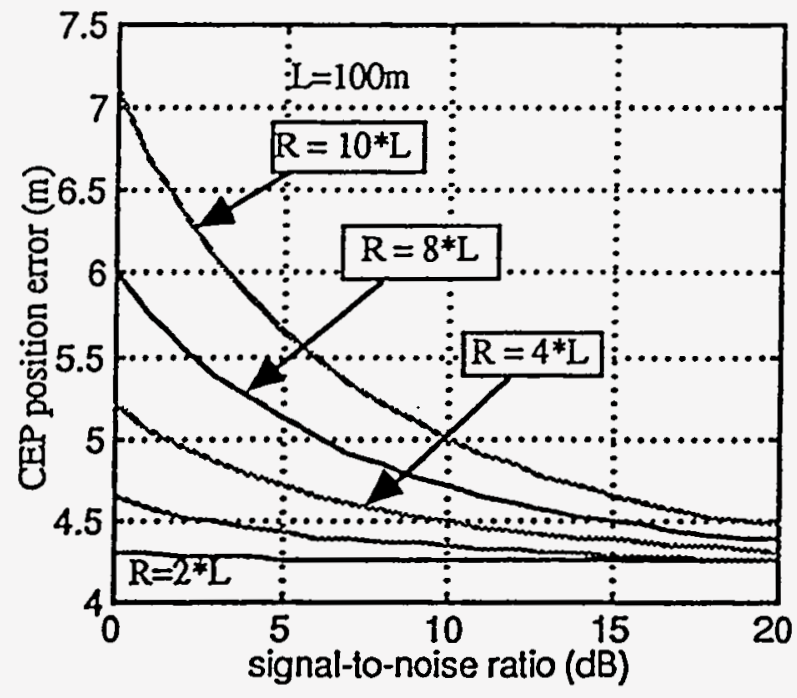

Figure 8 Position error for a simulation scenario

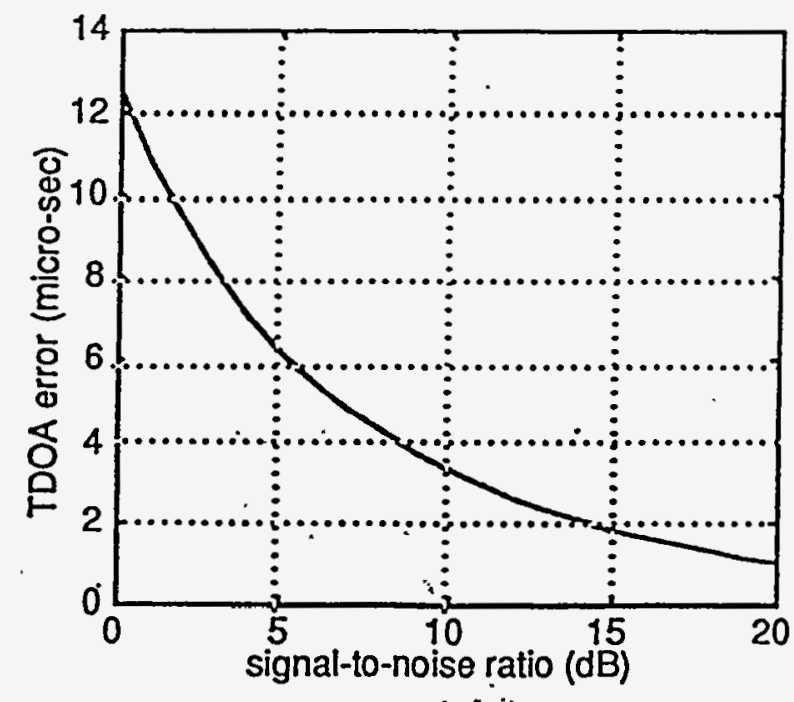

Figure 9 TDOA estimation error versus SNR

\section{Simulation Studies}

To demonstrate the localization ability from triangulation, we developed a digital beamformer and its associated signal processing function as shown in Figure 3. One can test and validate the beamformer software with modeled sensor input signals. Figures 10 and 11 show the results of the simulation. Here we assumed a sensor array geometry exactly as that chosen for the "Olney" experiment. We assumed a broadband signal consisting of a $100 \mathrm{~Hz}$ tone with a series of harmonics at $200 \mathrm{~Hz}, 300 \mathrm{~Hz}, \ldots$, up to $1 \mathrm{kHz}$ is 
located' at the origin of the coordinate system. We sampled the data at $3 \mathrm{kHz}$ as in the "Olney" experiment. Figures 10 and 11 each shows the array geometry, temporal time signal at one of the sensors, and cartesian and polar plots of the beamformer outputs. Array one shows the correct direction $\left(204^{\circ}\right.$ ) with respect to the $x$ (abscissa) axis. Array 2 also shows the correct direction of $176^{\circ}$ to the source.

Figure 12 shows that the two sensor arrays correctly identify and localize the source position by triangulation. Furthermore, by superpositioning the beam patterns onto the actual "Olney" map as shown in Figure 13, the two beams point to the exhaust stack as the source. This simulation study basically verified that the beamformer software is valid and produced a correct answer with simulated inputs.

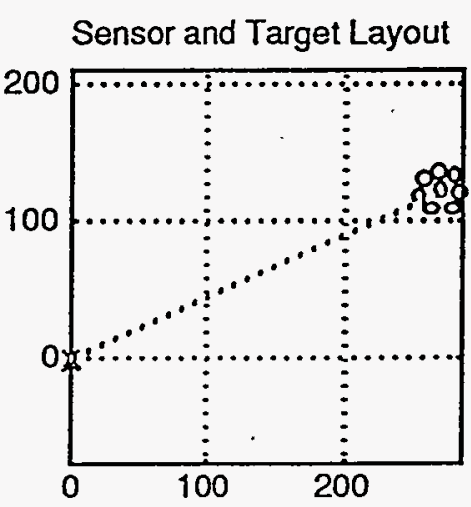

Received Signal Power

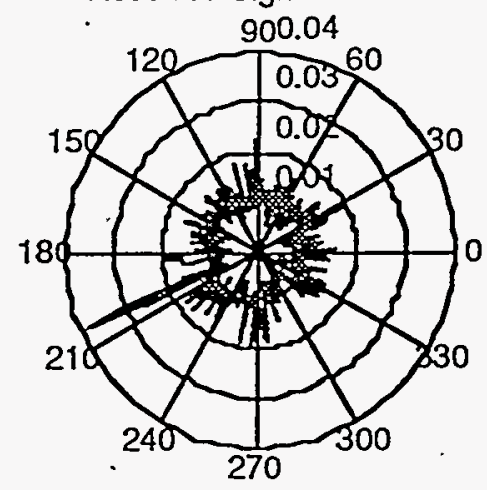

Source Signal vs. Time(sec)

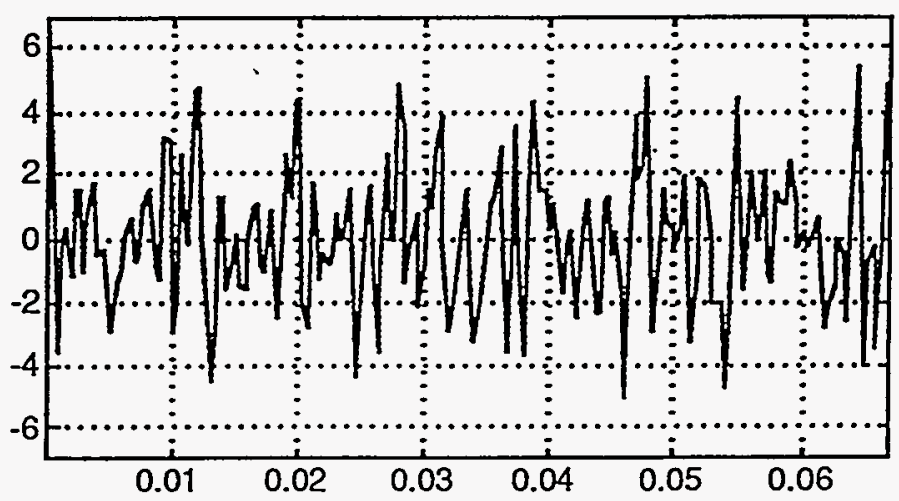

Received Signal Power vs. Beam Angle(degrees)

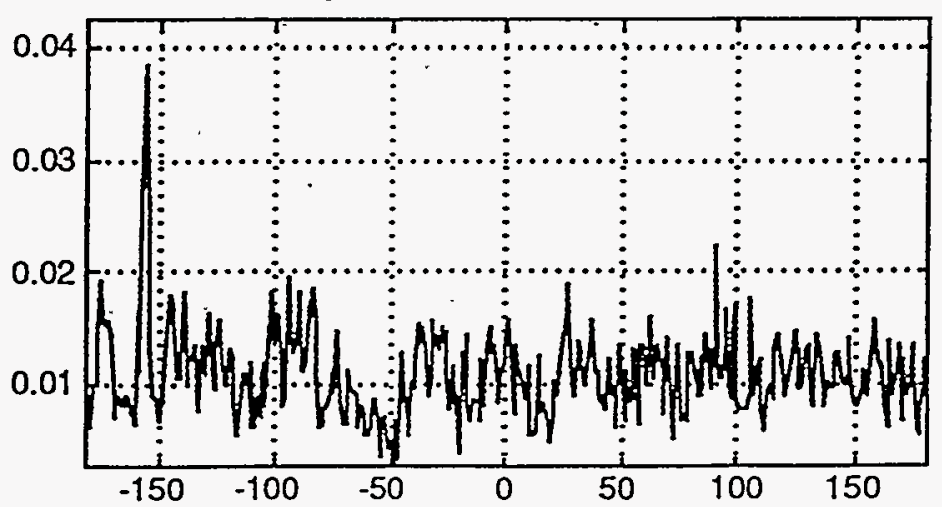

Figure 10 Beamforming simulation with an eight element circular array: array \#1 

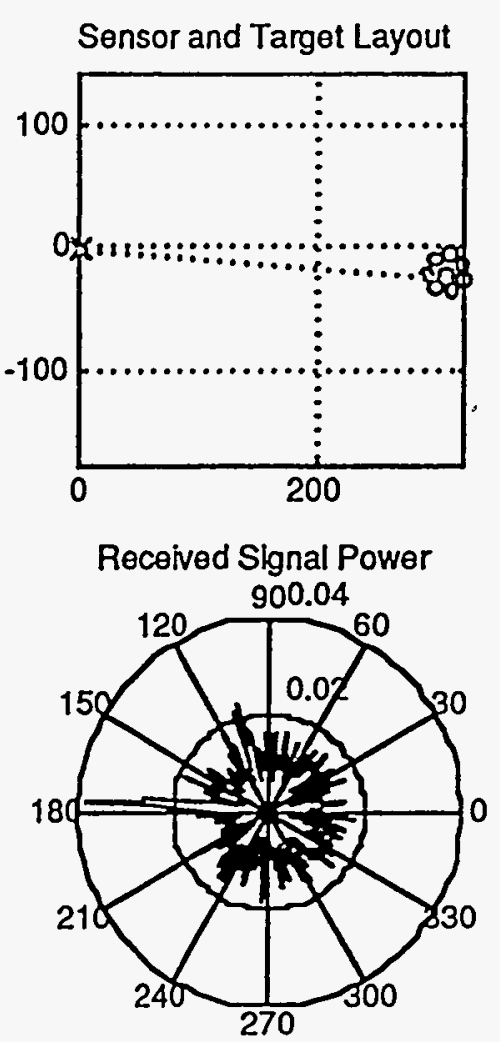

Source Signal vs. Time(sec)
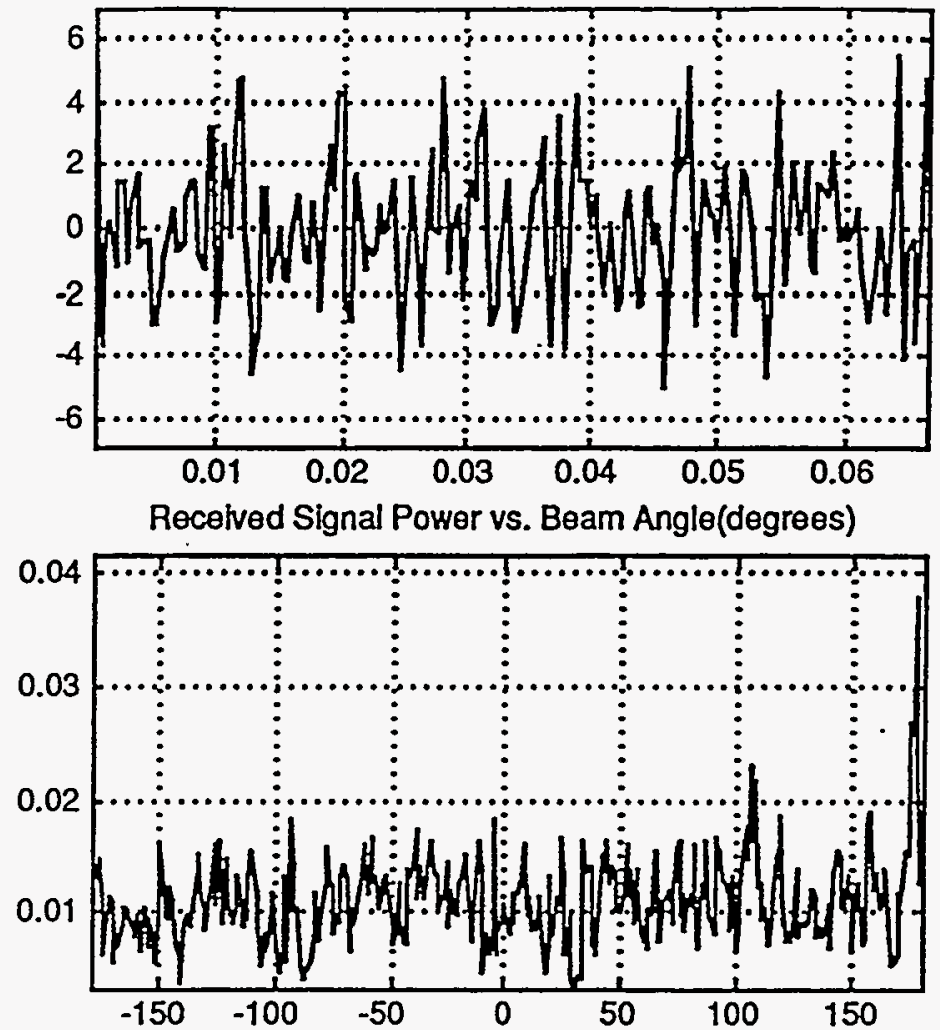

Figure 11 Beamforming simulation with an eight element circular array: array \#2

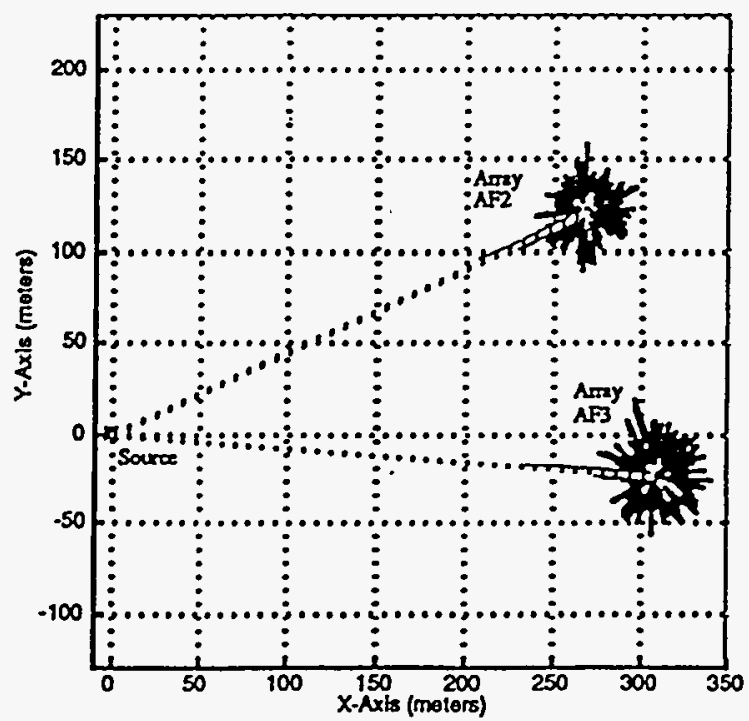

Figure 12 Two sensor arrays correctly triangulate to localize the source

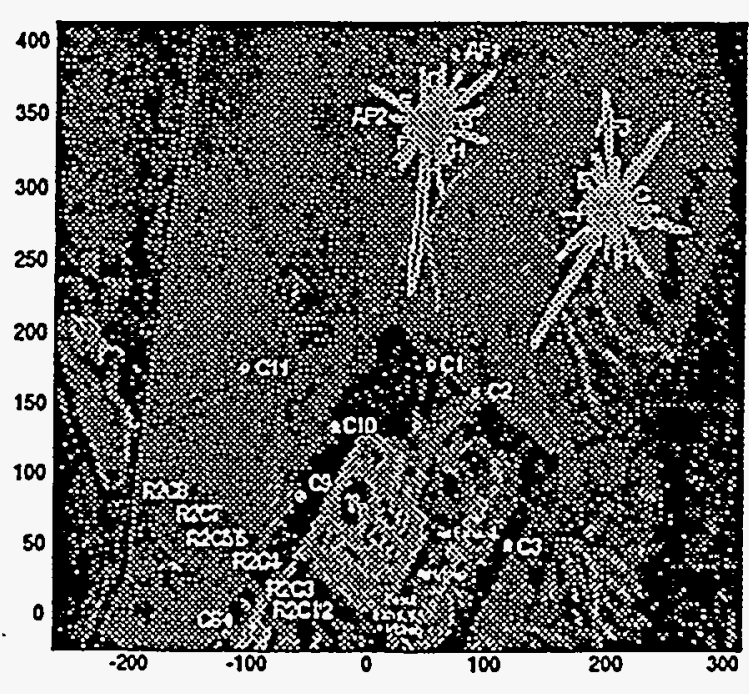

Figure 13 Superposition of simulated beam patterns on actual "Olney" geometry. 


\section{Analysis of LLNL onsite Acoustic Experiments}

Since we have validated the software on simulated data, we are interested in exercising the software with actual experimental data. Towards this end, we conducted several low cost acoustic experiments at LLNL's buffer zone area near the westgate entrance on August 7, 1995. Figure 14 shows the approximate map of the site. The site was a grassy area with tall eucalyptus trees lining the top (vertical is East), and low profile trees separating the field from the adjacent Vasco road traffic. The sound source was from a portable Honda generator and the 8 microphones were bought from a local Radio Shack store. We conducted a total of 10 experiments with different configurations of array geometry. Each experiment consisted of a generator on and generator off event. We also moved the source in a square pattern to test localization performance. For example, we collected data from an equally spaced linear array, an optimally spaced linear array, a circular array and two subarrays with a separation baseline of 50 feet. The site was not the best in terms of having a large open space and quiet ambient environment because of wind noise, reflection from the trees, and vehicle traffic. Furthermore, since the local airport is less that 5 miles away, airplane traffic was also unavoidable. Although the site did not give us the best experimental conditions, however, it may actually be a more realistic environment.

Figure 15 shows a typical power spectral density (PSD) from two data segments: signal on and signal off. The signal off case can be equated to the ambient environment. It: can be seen that the signal contained a lot of narrowband tones on top of a broadband signature. Taking the ratio of signal power to noise power yields the SNR estimate as shown in Figure 16. This broadband signal was definitely related to the source and not due to. wind noise because when the source was turned off, the broadband signal also disappeared as shown in Figure 17, where we show the spectrogram near the source off time near the 11 second mark.

Figure 18 shows the beam pattern from a linear array. The maximum response direction is obviously pointing to the correct direction of the source. The generator was actually aligned with the the 3rd microphone from the top. With the source at 50 feet and a 2 foot element spacings, we predicted that the the source is at $3^{\circ}$ left of the array axis. This is in good agreement with the result. Aside from the main lobe, there are several minor lobes that seem to indicate the existence of other sources of interference. The interference sources are notably coming form vehicle traffic below and tree reflections from the top. In fact we believed reflections from the trees were the main contributor of the broadband signal we detected.

Figure 19 shows the beam pattern from a circular array. Again the main lobe is pointed in the correct direction. The source direction is about $10^{\circ}$ off from what we expected. Since the microphones were laid without using a precision surveyed instrument, we trully expect the array axis of the circular may be off as much as several degrees. The $10^{\circ}$ may also be caused in part by interference sources. 


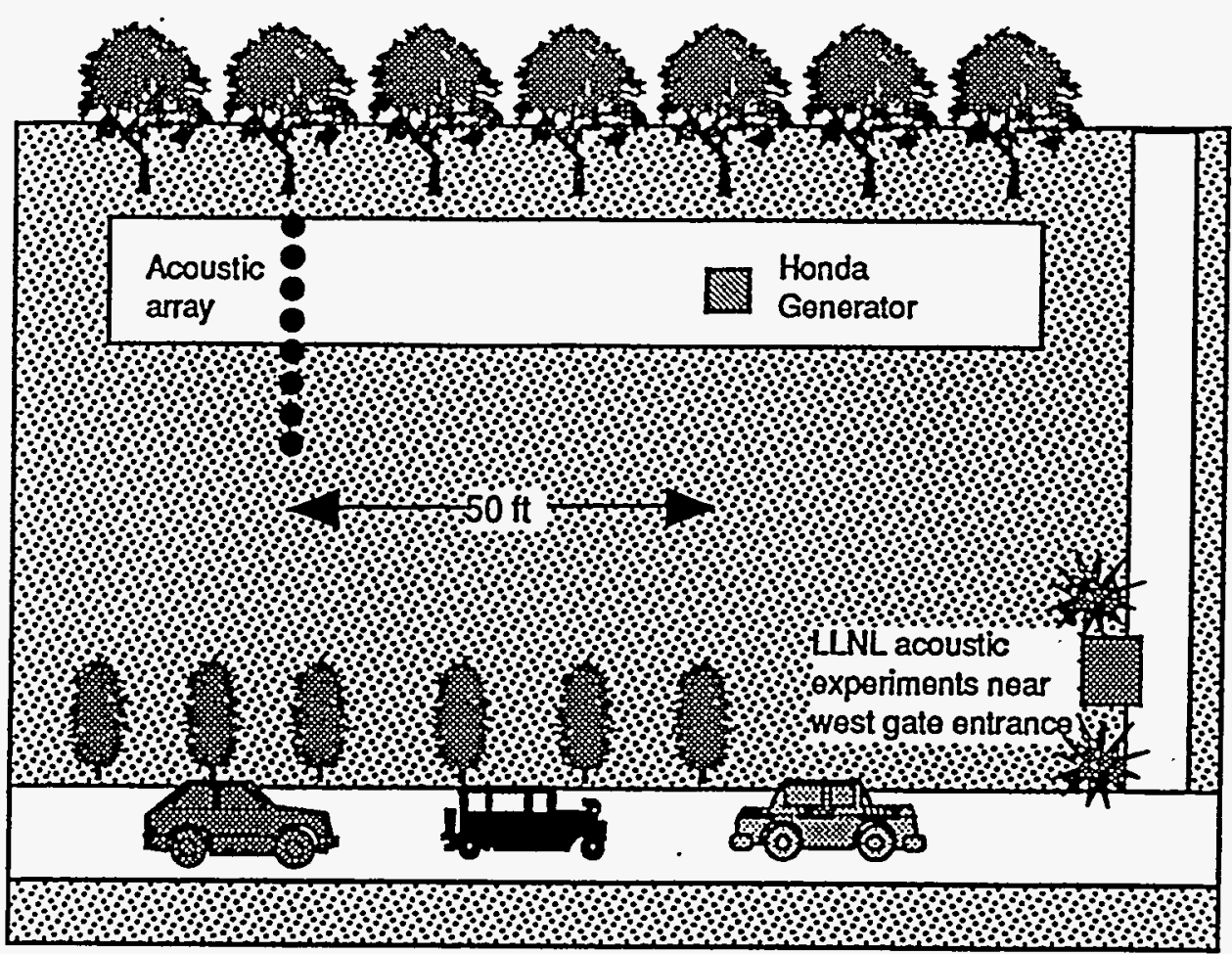

Figure 14 Schematic map of LLNL acoustic experiment site
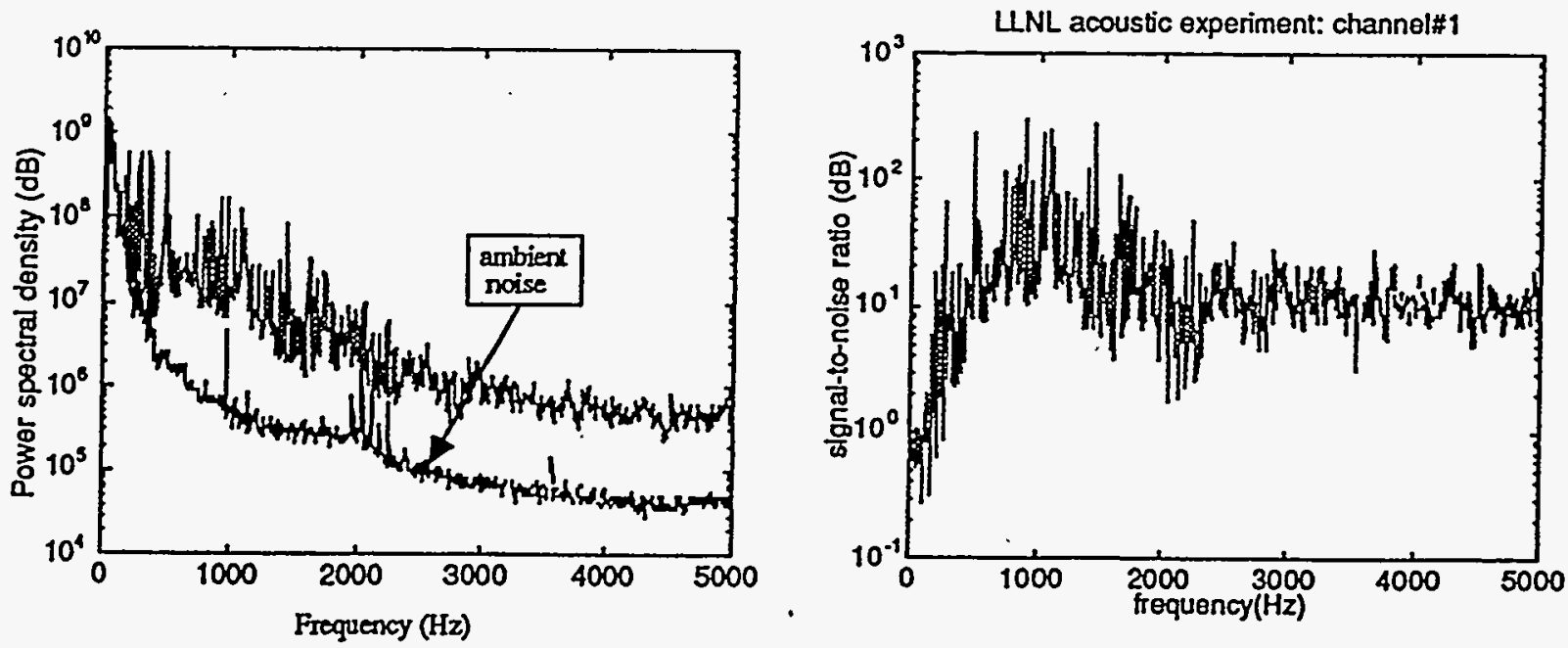

Figure 15 Power spectrum of signal and noise. Figure 16 Signal-to-noise ratio versus frequency 


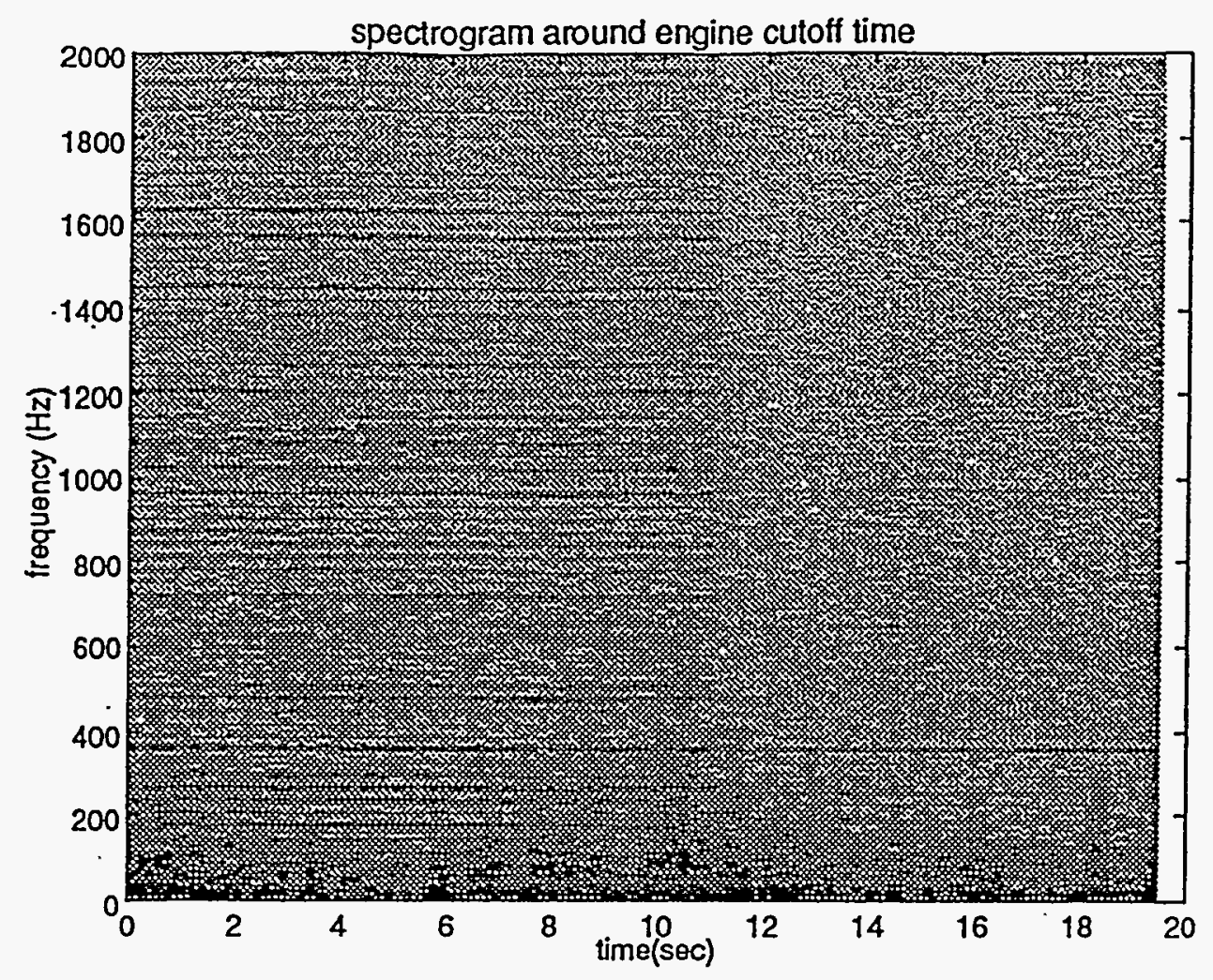

Figure 17 Spectrogram of sensor output near source off time at the 11 second mark.

$\operatorname{T1ONCH}(1,3-8), b w=10-200 \mathrm{~Hz}, v=340 \mathrm{~m} / \mathrm{s}$

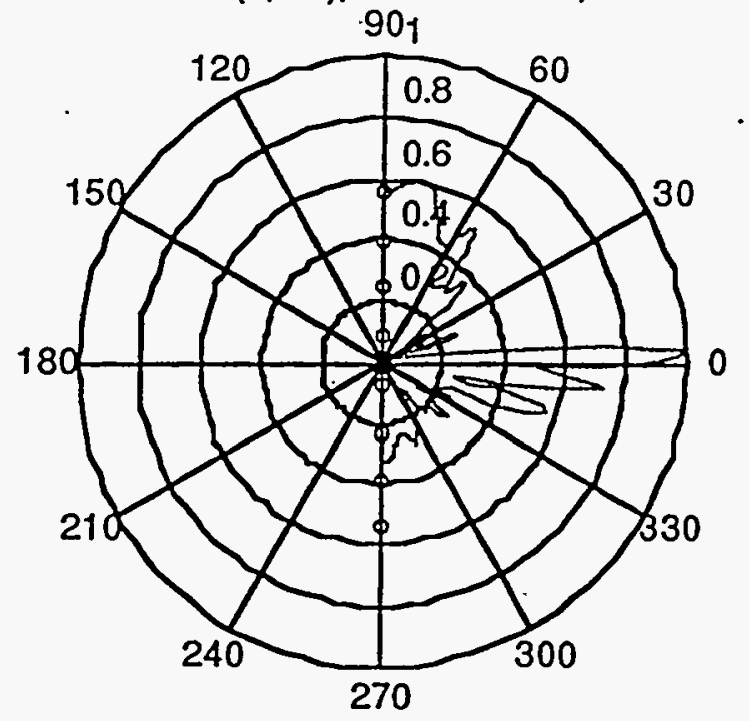

T4ONCH(1,3-8), bw=10-200Hz, v=340m/s

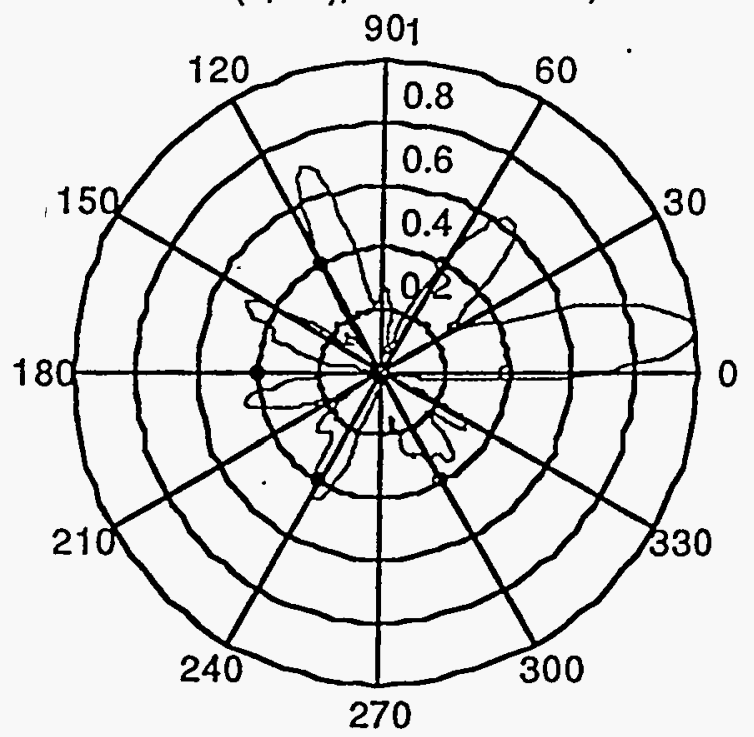

Figure 18 Beam pattern from a linear array

Figure 19 Beam pattern from a circular array 


\section{Analysis of Olney Experiments}

As part of the TUGS project, Olney experiments were conducted in early July of 1995. Signal sources are from three diesel generators in an underground structure. Geophone arrays were deployed as shown in the map in Figure 20. Sensor locations from the original data were given in a left-handed coordinate system. For convenience, we have transformed it to a right-handed coordinate system. The $X$ axis is pointing towards array AF3 and is along the edge of the parking lot. The $\mathrm{Y}$ axis is $90^{\circ}$ from the $\mathrm{X}$ axis and is along the other edge of the parking lot toward the parking lot entrance. Table 1 below gives the sensor locations:

\begin{tabular}{|l|l|l|l|}
\hline Circular array & $X(\mathrm{~m})$ & $\mathrm{Y}(\mathrm{m})$ & $\mathrm{Z}(\mathrm{m})$ \\
\hline \hline AF2A & 269.70 & 121.43 & 9.45 \\
\hline AF2B & 278.40 & 108.93 & 9.89 \\
\hline AF2C & 284.53 & 120.25 & 10.08 \\
\hline AF2D & 280.40 & 132.19 & 9.65 \\
\hline AF2E & 269.02 & 137.00 & 9.01 \\
\hline AF2F & 257.72 & 131.02 & 8.56 \\
\hline AF2G & 255.03 & 117.46 & 8.66 \\
\hline AF2H & 264.05 & 107.56 & 9.15 \\
\hline AF3A & 308.75 & -23.32 & 6.75 \\
\hline AF3B & 312.75 & -37.61 & 7.42 \\
\hline AF3C & 322.01 & -29.81 & 8.43 \\
\hline AF3D & 321.90 & -16.02 & 8.96 \\
\hline AF3E & 310.39 & -8.06 & 8.44 \\
\hline AF3F & 298.49 & -11.89 & 7.10 \\
\hline AF3G & 293.69 & -22.47 & 5.75 \\
\hline AF3H & 299.27 & -34.65 & 6.20 \\
\hline
\end{tabular}

Table 1 Olney circular array surveyed locations in a right-handed coordinate system

The Olney data were collected with a series of signal on and signal off experiments. There were all together five different sessions. Our goal here was to localize the source via triangulation from the two sensor arrays. Since the baseline between arrays is known, we need to find the source bearings from the sensor arrays. We apply the same technique as before to process the Olney data. The generator is known to have a stable signal at about 20 $\mathrm{Hz}$.

Figure 21 shows the full spectrogram of a single channel output for experiment af2-1, where the signal was turned off at 60 seconds. We saw strong spectral lines at 6,9 , and 13 $\mathrm{kHz}$ for all time. However these could not be the true signals because the signal was off at 60 seconds. It was suspected that these signals came from the data digitization process. Therefore valid signals are near $100 \mathrm{~Hz}$, and seem to disappear just before the 60 second mark. Figure 22 shows a cut spectrogram of the same plot but focused at frequencies below $350 \mathrm{~Hz}$. Note the strong signal that starts at $100 \mathrm{~Hz}$ but decayes to about $80 \mathrm{~Hz}$ steady state in 30 seconds. This signal, exhibting a strong doppler shift, is from the overhead passing of an airborne vehicle. We could not find the $20 \mathrm{~Hz}$ signal in this channel.

Figure 23 shows the beamformed result. Since we know the source location, we beamformed the array to the source direction. Again spectral plot of the beam data failed to indicate the presence of the $20 \mathrm{~Hz}$ data. 
In order to investigate the true presence of the source signal, we took two 10 second segments of the data: from 10 to 20 seconds when the generator was on, and 70 to 80 seconds when the generator was off. We computed the noise (generator off) power spectrum and used it to normalize (or pre-whiten) the generator on power spectrum. The result is the signal-to-noise ratio that gives strong indication of the presence of the true signal. Figure 24 shows the results of this operation. The result shows that indeed the strongest signal came from the decaying $100 \mathrm{~Hz}$ tone. It was measured at $86 \mathrm{~Hz}$ at 20 seconds from measurement start. This signal is $10 \mathrm{~dB}$ higher than the background noise.

In order to form beams we need to know the propagation speed. For a seismic data, the speed could vary by a factor of three dependent on the type of wave motions [11]. We resolve this problem by computing the cross correlation function of each sensor with respect to the center element. By measuring the relative time delay between two sensors we can compute the speed of propagation. Figure 25 shows the resulting computations. Our estimate of the time delay between sensors \#7 and \#4 is about $100 \mathrm{~ms}$, and the sensor spacing is about 30 meters, so the propagation speed is about $300 \mathrm{~m} / \mathrm{s}$.

Choosing a signal bandwidth of 75 to $125 \mathrm{~Hz}$, we beamformed the two sensor arrays by steering the array at a one degree increment. The results are shown in Figure 26 . The resulting beam patterns do not seem to give a clear direction to the true source location. Perhaps the signal to noise ratio is too low. A better result may be achievable with a narrower processing frequency band. In addition, a significant number of grating lobes was observed due to the undersampling of the array at this frequency.

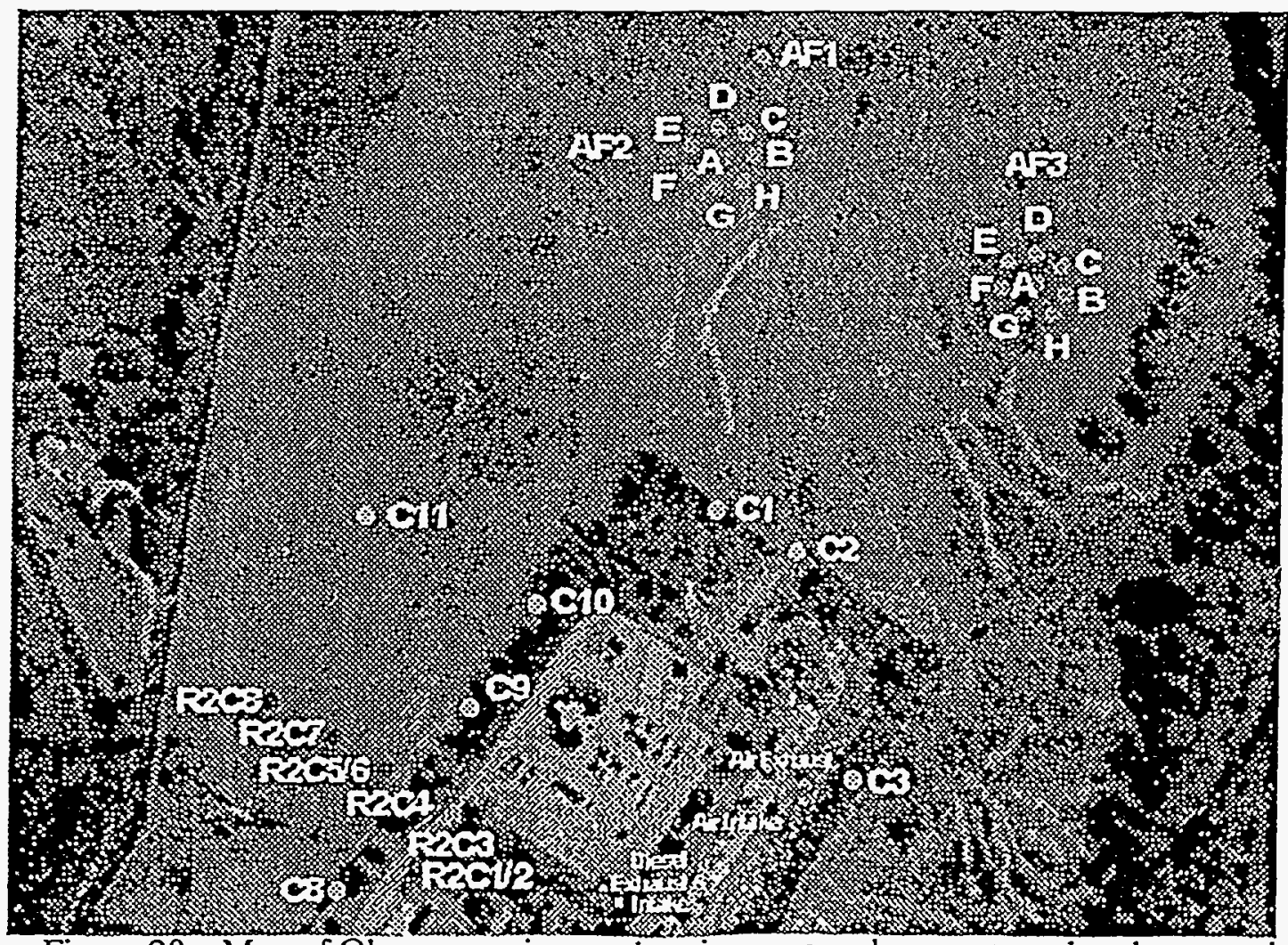

Figure 20 Map of Olney experiment showing sensor placements and underground structure. 


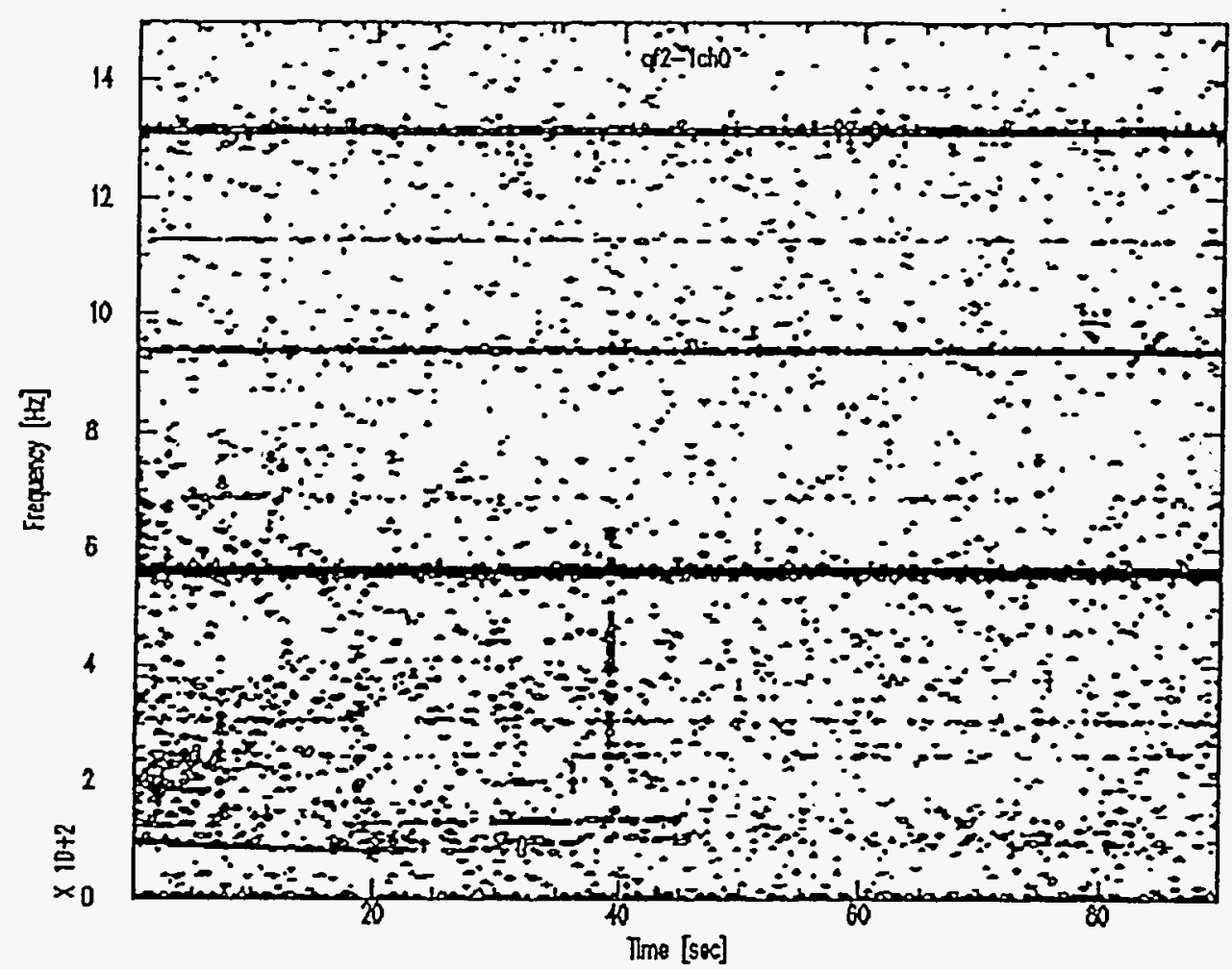

Figure 21 Full spectrogram of a single channel data from experiment af2-1ch0

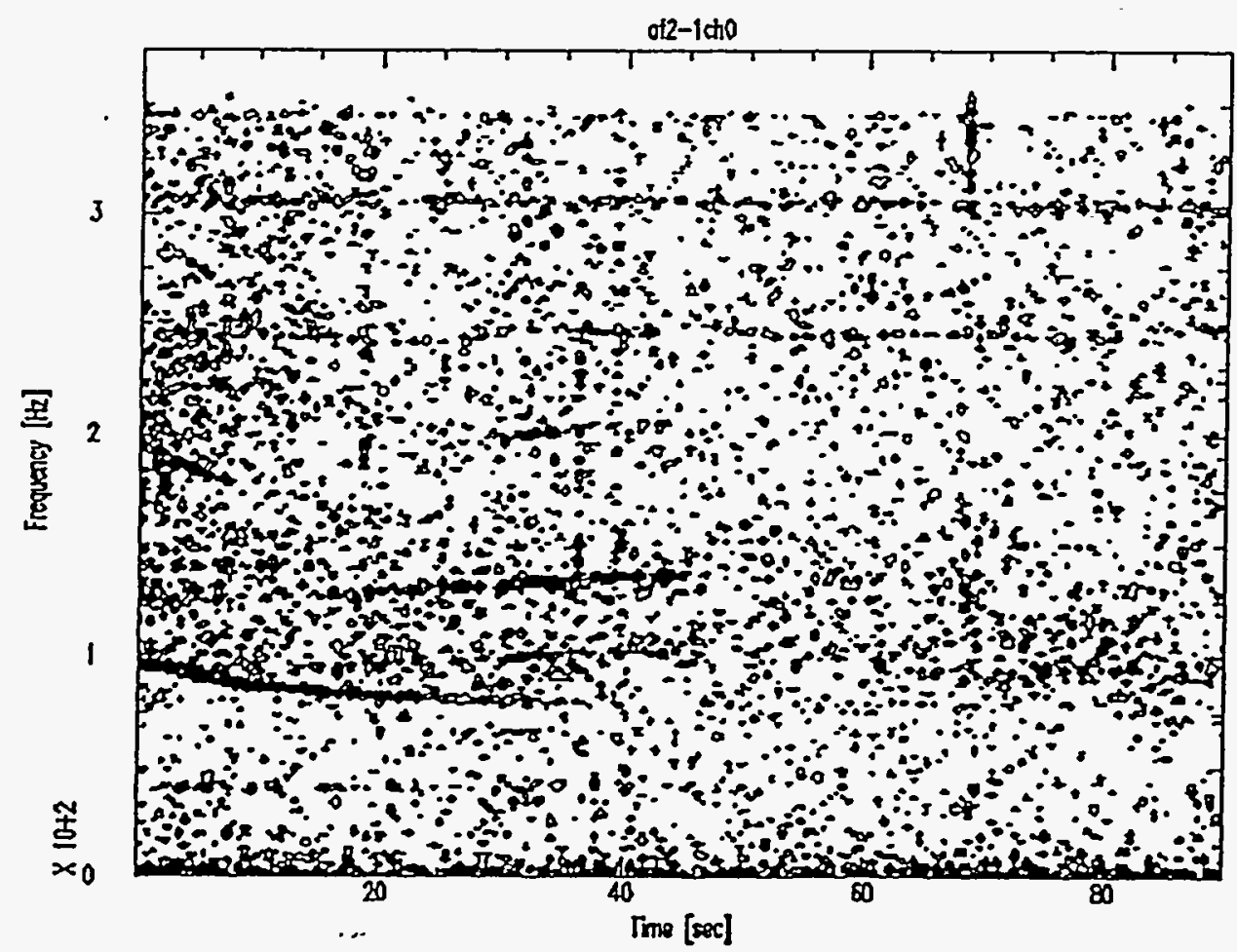

Figure 22 Cut spectrogram of a single channel data from experiment af $2-1 \mathrm{ch} 0$ 


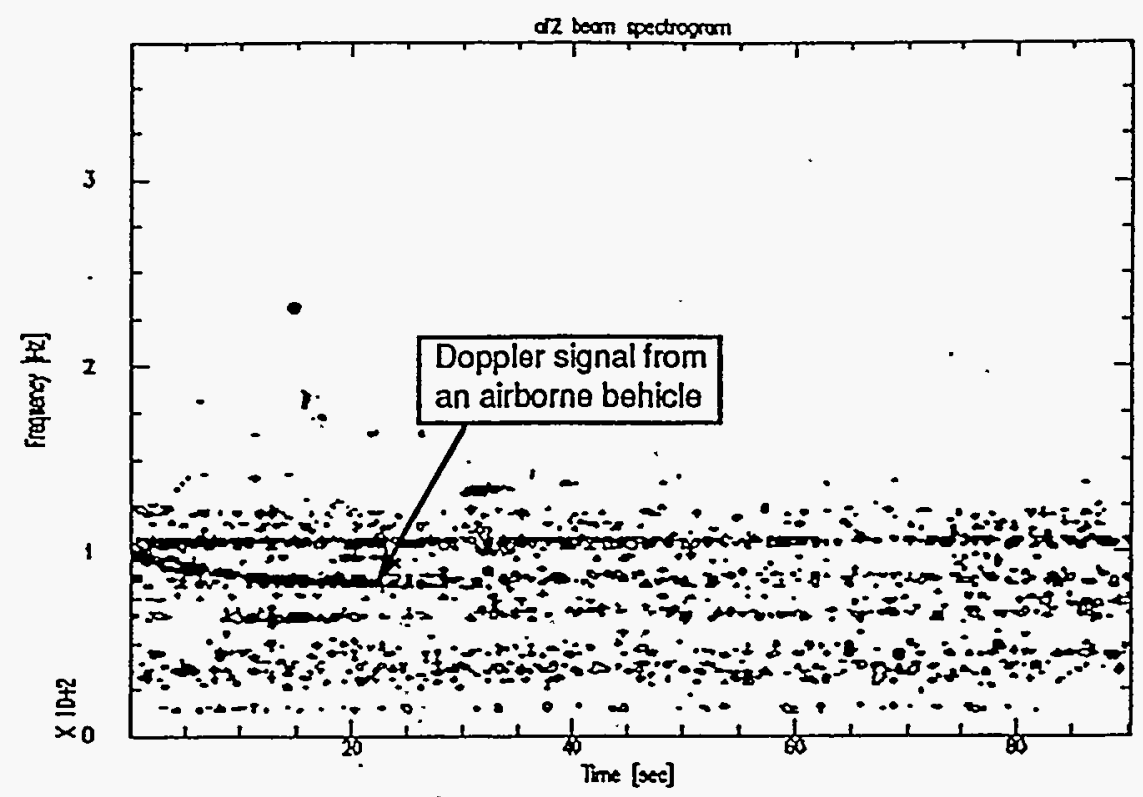

Figure 23 Gyt spectrogram of a full beam data from experiment af2-1
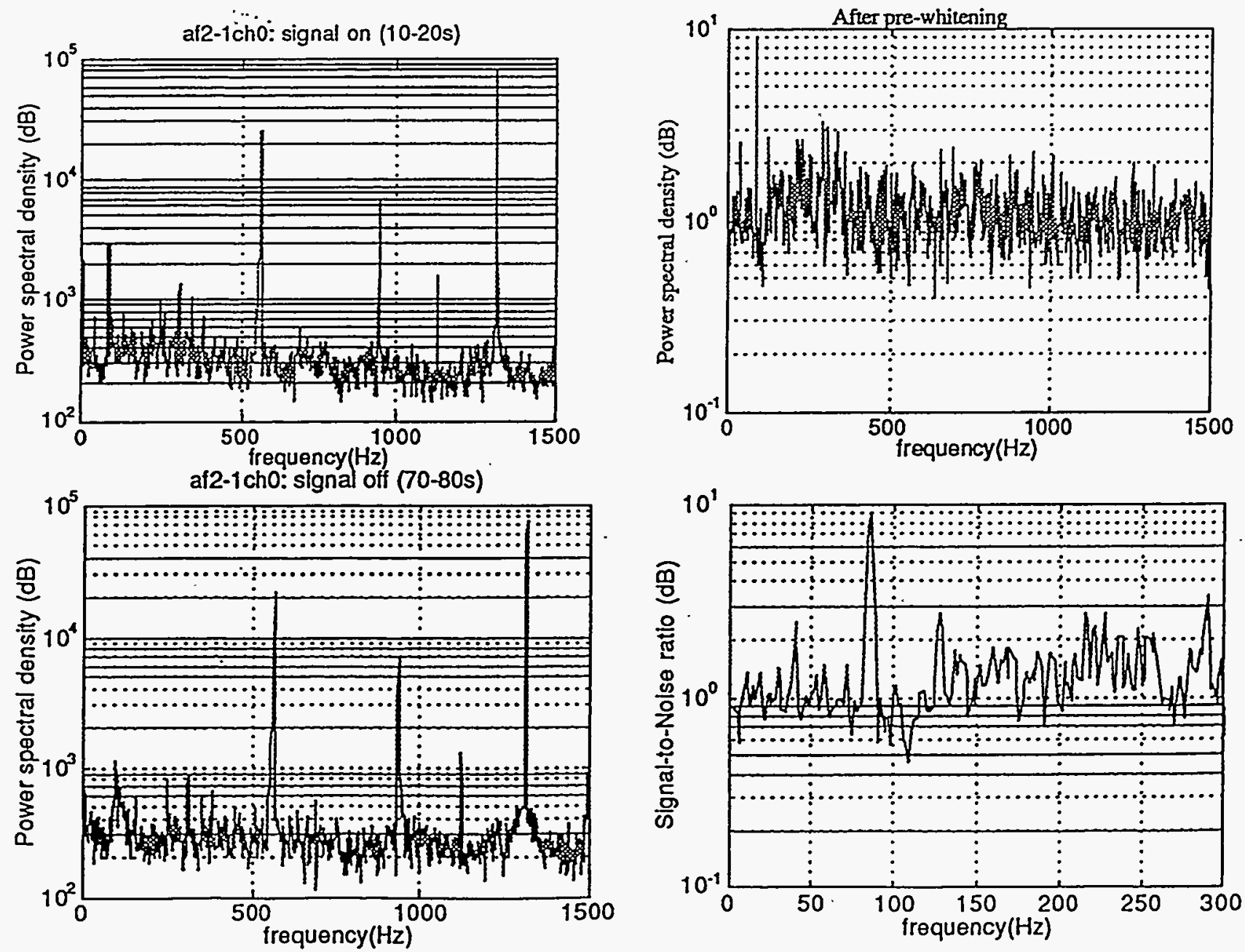

Figure 24 Signal detection from a single channel 


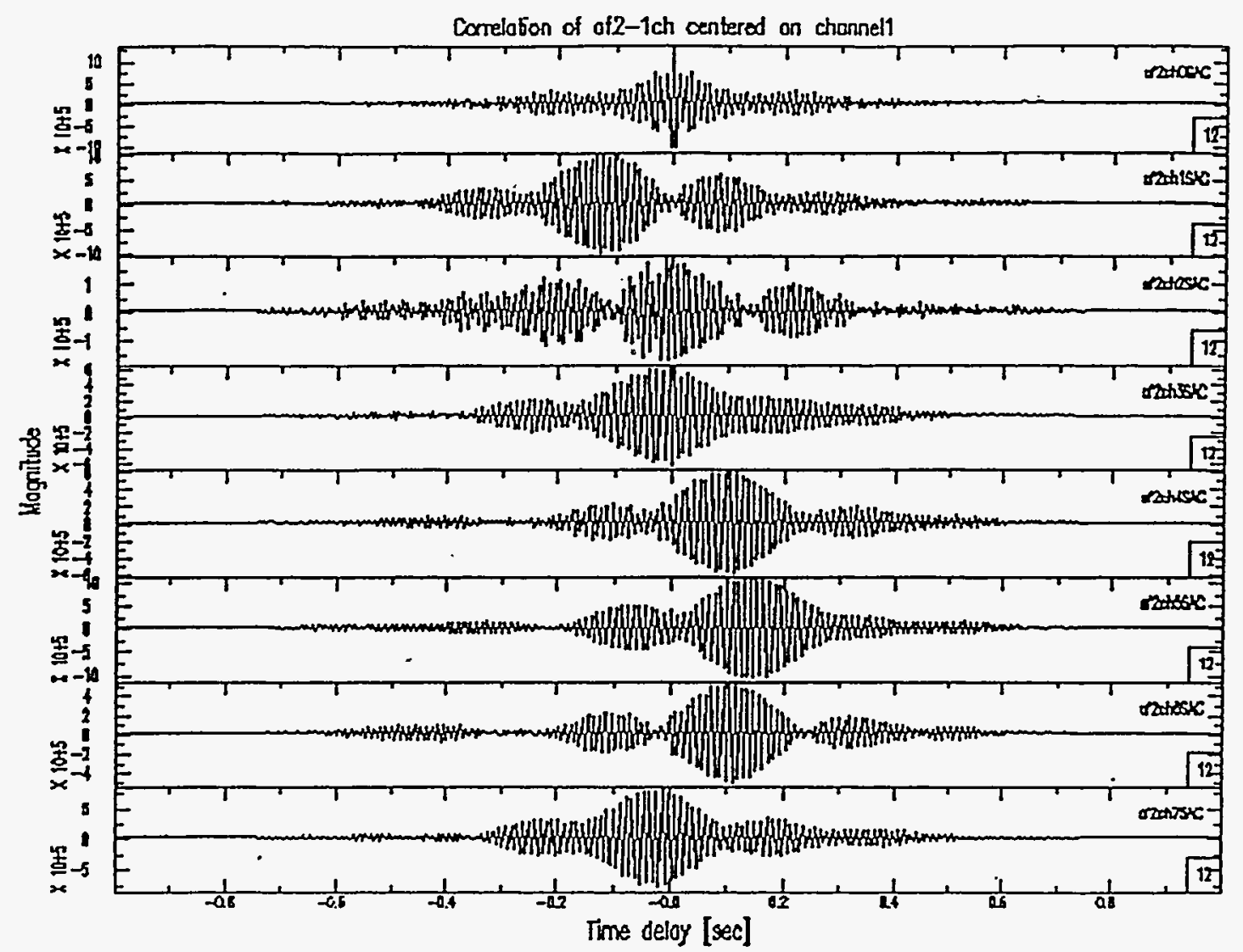

Figure 25 Multisensor correlation from experiment af2-1. Top trace is the autocorrelation of channel 1, bottom trace is the cross-correlation between channel 8 and channel 1.

Figure 26 shows similar analysis of antenna \#3 data. However the $20 \mathrm{~Hz}$ signal can be found in almost al the channels. The signal cut off at 60 second is clearly observable. It is to be expected that signal level should probably vary among channels in relation to their distance to the source. Figure 27 shows the power spectral density of all 8 channels from 0 to $30 \mathrm{~Hz}$. We measured the signal levels and plotted them against the sensor locations with respect to the source. The results are shown in Figure 28. We saw a reduction of $14 \mathrm{~dB}$ over a distance of $30 \mathrm{~m}$. This is significantly greater than expected for an inverse square loss assumption. For example, with a closer sensor at $295 \mathrm{~m}$ and the further at $325 \mathrm{~m}$, the loss is no greater than $7 \mathrm{~dB}$. Also the signal levels do not dropped monotonically as a fuction of distance from the source. A possible explanation may be that the sensor gains are not identical or that interface between the sensor and the ground has uneven attenuation.

Figure 29a and 29b shows the resulting beam patterns formed from the 8 sensor arrays. For array \#3 we found the the propagation speed is about $500 \mathrm{~m} / \mathrm{s}$ using the multisensor correlation proceedure similarily computed for array \#2. We concluded that array \#2 does not give a good indication of the source direction, while array \#3 gives an approximate direction to the source. From the geometry, the correct source directions for array \#2 and Array \#3 are $207^{\circ}$ and $175^{\circ}$ respectively. The actual measured directions from the beam patterns are $255^{\circ}$ dnd $170^{\circ}$ respectively. Pictorially, we superimpose the beam patterns on the actual Olney site location as shown in Figure 30. 

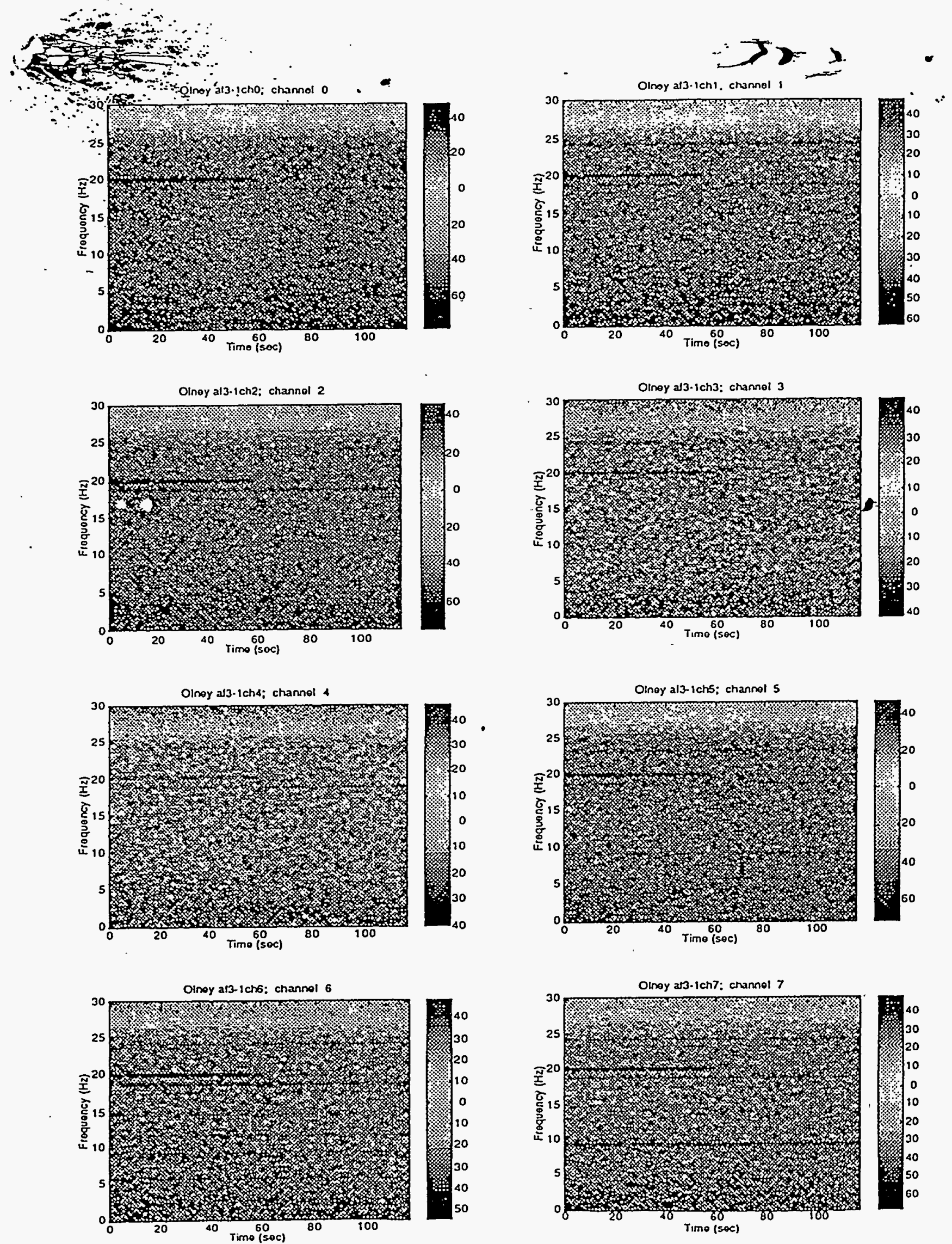

Figure 26 Detection of a $20 \mathrm{~Hz}$ signal on antenna \#3 

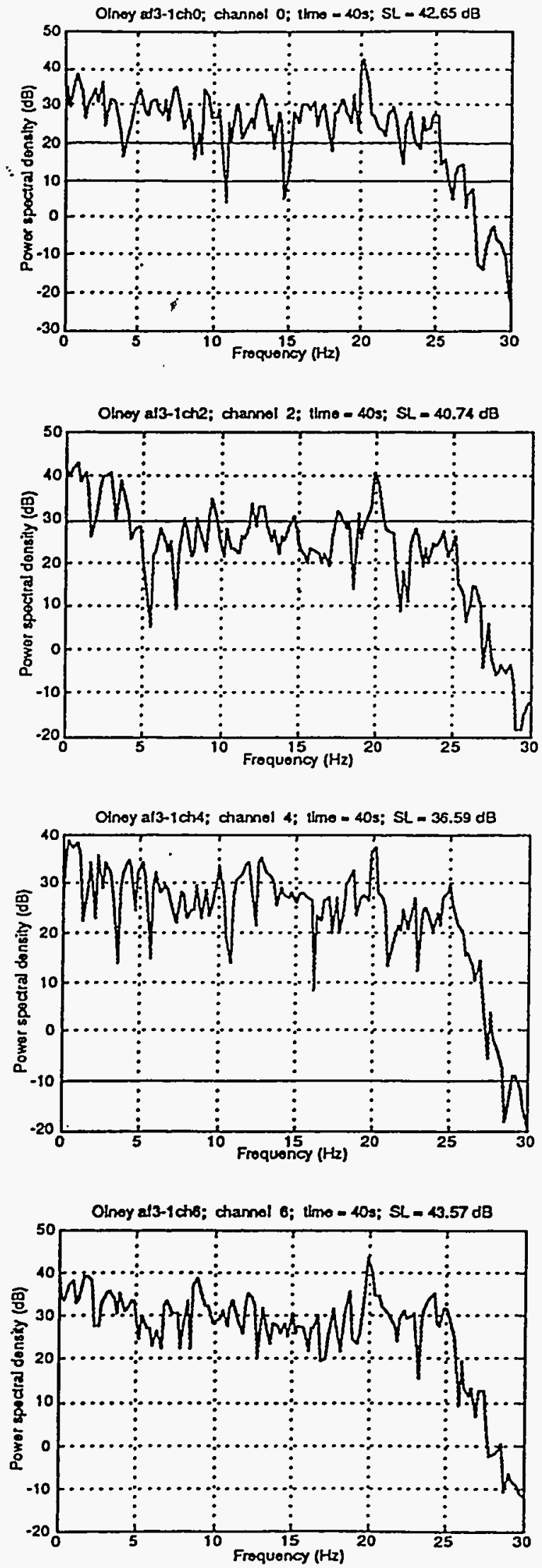
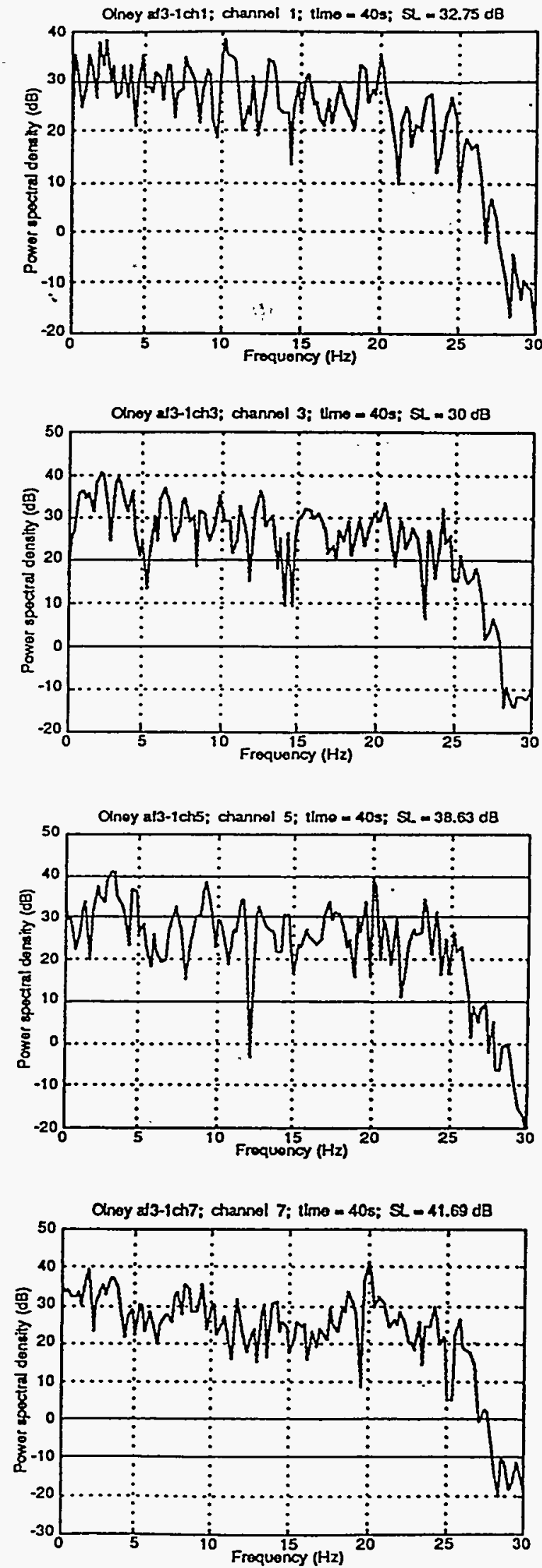

Figure 27 Power spectral density of signal across all 8 channels 


\section{Olney af3-1}

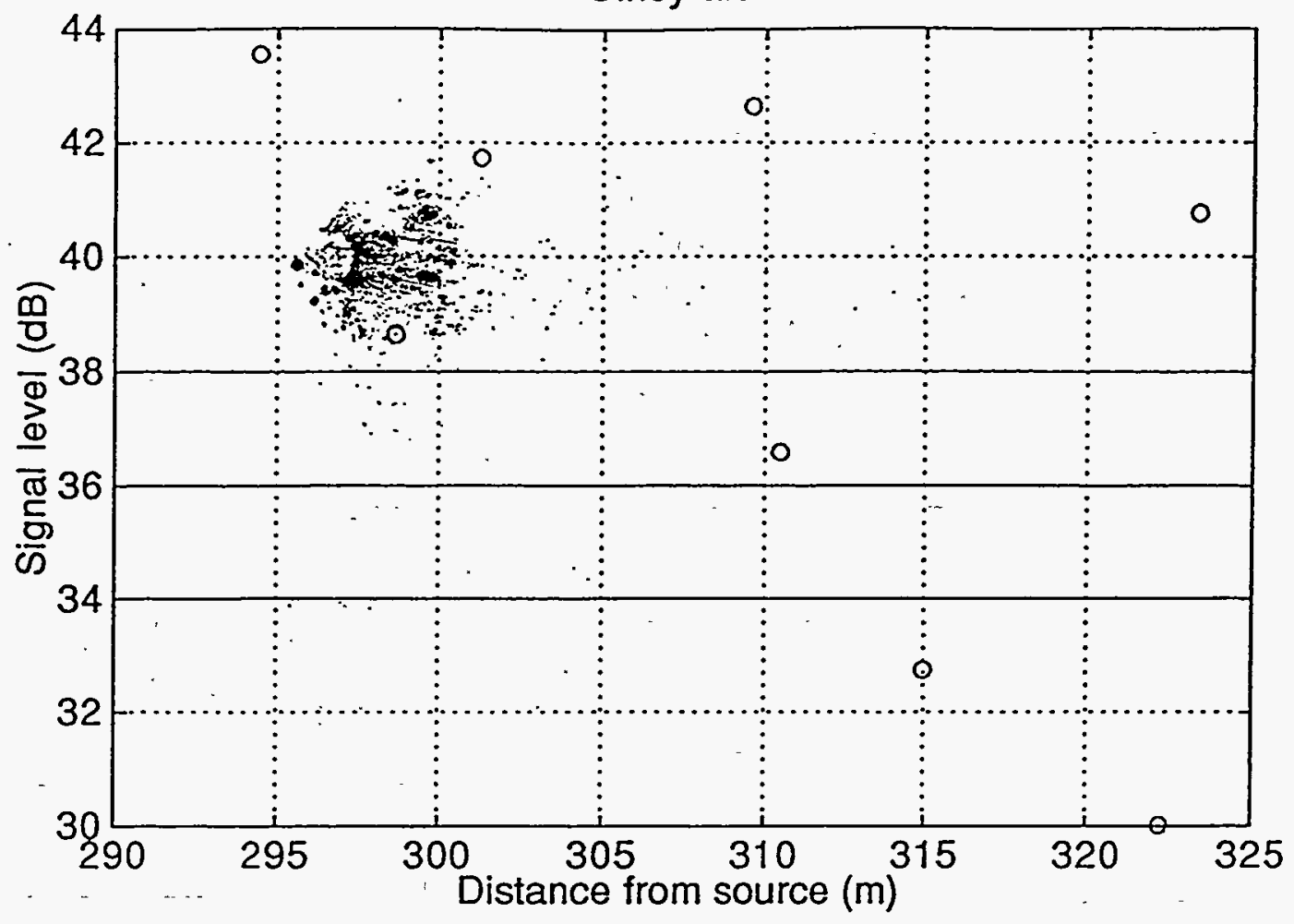

Figure 28 Signal levels across all channels for antenna \#3

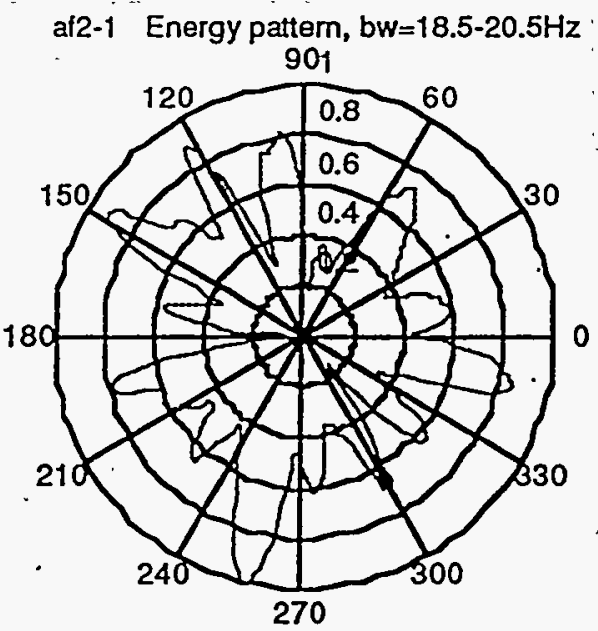

Figure 29a Beam pattern for antenna \#2

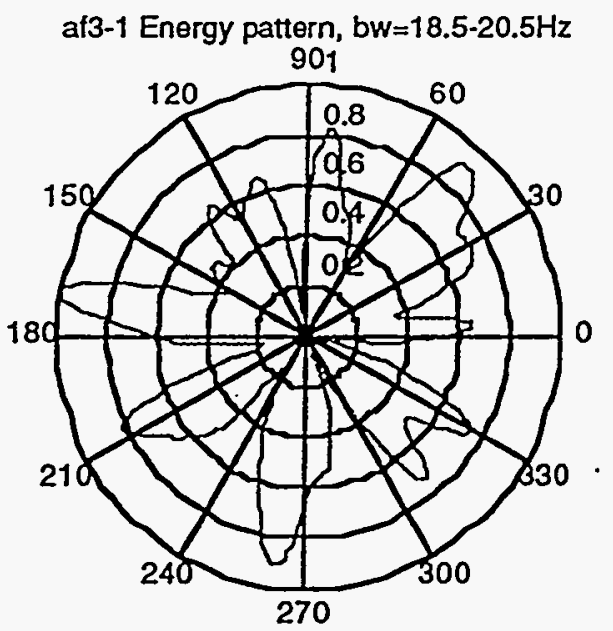

Figure 29b Beam pattern for antenna \#3 


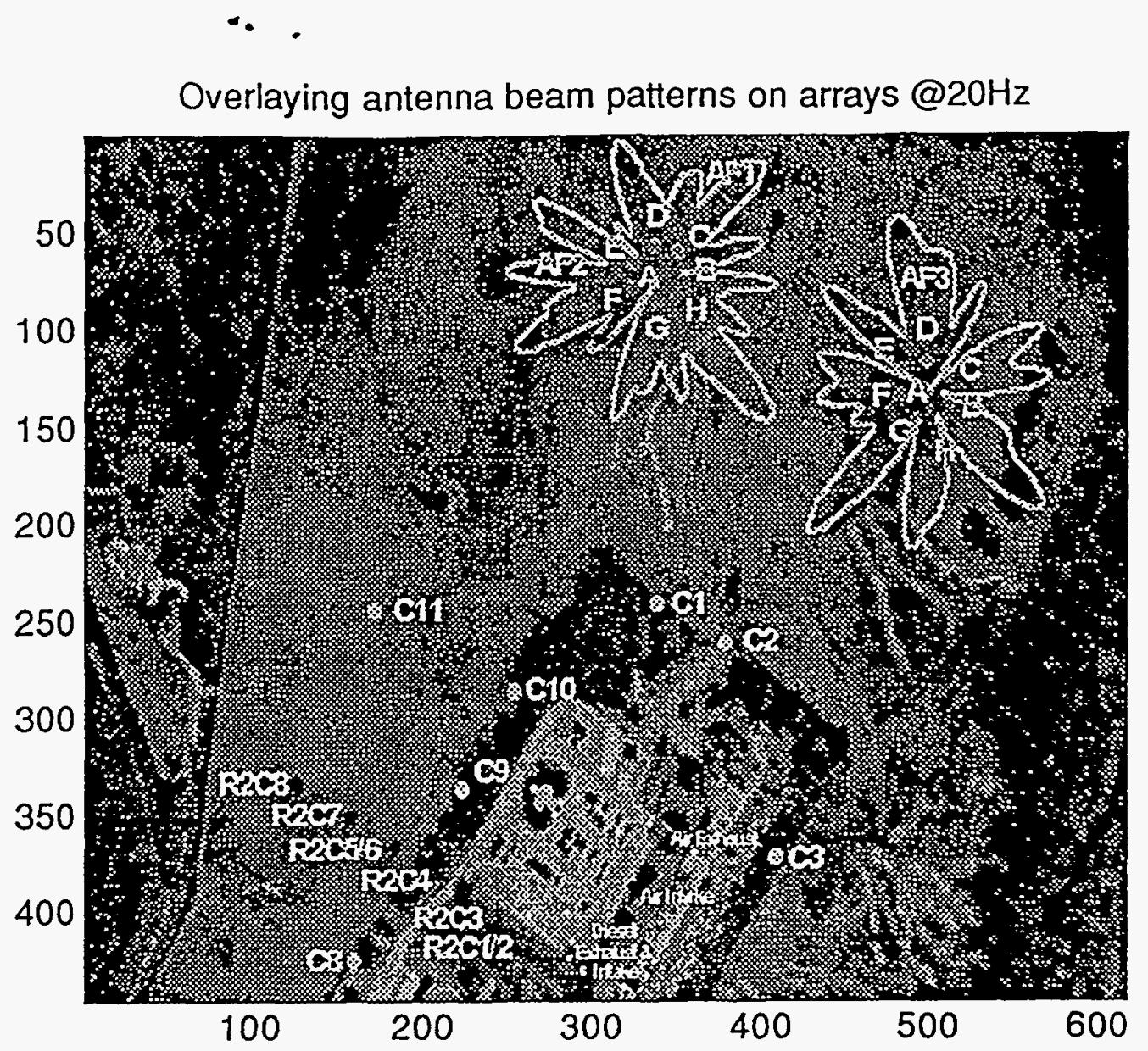

Figure 30 Beamforming results from Olney data and superposition onto the true map location.

\section{Summary and Conclusions}

We have developed software to process and analyze data from unattended ground sensors. We have validated our methodology, through simulation, and further verified it with acoustic experiments conducted at LLNL. We obtained good predictions of source location with respect to the ground tnuth. We observed that the LLNL experiments were conducted near a realistic environment with wind noise, vehicle traffic, air traffic, and other sources of interferences.

We applied the same technique to analyze the Olney data. However our results are inconclusive. We failed to detect the signal on array \#2 although we found it on array \#3. The beam direction of array \#3 indicated an approximate direction to the source to within $5^{\circ}$. The error may be the result of uneven sensor gains due to either sensor calibration or. ground placement. 


\section{References}

[1] P. Pearson,"RSP Data Fusion for Internetted Unattended Ground Sensors Program," A proposal to ARPA BAA N66001-94-X-6057, October 27, 1994.

[2] T. Logsdon, The Navstar Global Positioning System, Van Nostrand Reinhold, 1992.

[3] R.O. Nielsen, Sonar Signal Processing,Artech House, 1991.

[4] S.U. Pillai, Array Signal Processing.Springer-Verlag, 1989.

[5] R.V. Nostrand,Seismic Filtering, Society of Exploration Geophysicists, 1971.

[6] J. Capon,"High-Resolution Frequency-Wavenumber Spectrum Analysis," Proceedings of the IEEE, Vol. 57, N0. 8, August 1969.

[7] J.P. Burg," Maximum entropy spectral estimation," in Proc. 37th Meet. Soc. Explor. Geophyscists, 1967:

[8] S.A. Hovanessian, Introduction To Sensor Systems, Artech House, 1988.

[9] L.C. Ng,"Multisensor Multitarget Time Delay Vector Estimation," IEEE Trans. Acoust. Speech, Signal Processing, ASSP Vol.34 (4), August 1986.

[10] R.W. Fidler and P.N. Carlton,"Locating Seismic Sources Passively," IEEE 1981 Proceedings of the 2nd International symposium on Computer-aided Seismic Analysis \& Discrimination.

[11] J.E. White, Underground Sound: Application of seismic wave, Elsevier Sciences Publishers, Netherlands, 1983. 
